\title{
Crystal structure and magnetic properties of $\mathrm{CuSb}_{2} \mathrm{O}_{4}$
}

\section{T. Atanasova · A. M. Strydom · C. J. H. Schutte ${ }^{\dagger}$ - L. C. Prinsloo · W. W. Focke}

Abstract The crystal structure of a copper antimonite $\left(\mathrm{CuSb}_{2} \mathrm{O}_{4}\right)$ was determined from X-ray powder diffraction data. The structure was solved by simulated annealing (SA) in direct space using the Rietveld method. The compound crystallizes in tetragonal symmetry, space group $P 4_{2} b c$ (106); unit cell parameters $a=b=8.76033$ (5) $\AA, c=5.79786$ (4) $\AA, Z=4, V=444.947$ (5) $\AA^{3}$ and density (calc.) $=5.539 \mathrm{~g} \mathrm{~cm}^{-3}$. The $\mathrm{CuO}_{6}$ polyhedra are strongly elongated due to Jahn-Teller distortion in a $[2+2+2]$ coordination arrangement, i.e. there are two long axial $\mathrm{Cu}-\mathrm{O} 1$ bonds of $2.447(13) \AA$, in the equatorial plane there are two intermediate $\mathrm{Cu}-\mathrm{O} 2$ bonds of 2.07(3) $\AA$ and two short $\mathrm{Cu}-\mathrm{O} 2$ bonds of 1.88(2) $\AA$. The $\mathrm{SbO}_{3}$ pyramidal arrangement is almost regular with Sb-O1 bonds of 1.97(2) $\AA(2 \times)$ and Sb-O2 of 1.959(5) A. The experimentally obtained Raman spectrum is consistent with values obtained from theoretical modelling studies. The magnetic behaviour of this new compound suggests that it belongs to the class of $S=1 / 2$ Heisenberg chain systems.

Keywords: Copper antimonite; X-ray diffraction; Crystal structure; Modelling; Raman spectroscopy; magnetic properties

\author{
M. T. Atanasova \\ Council for Geoscience, 280 Pretoria Road, Private Bag X112, Pretoria, South Africa
}

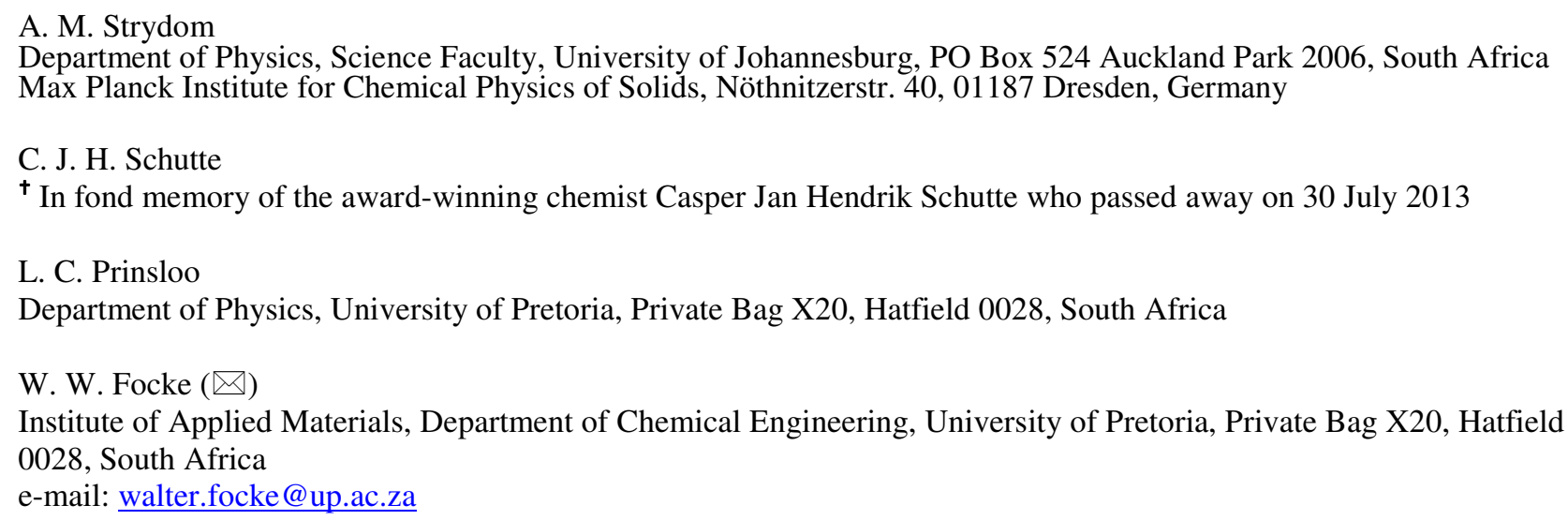




\section{Introduction}

The reactions and products in the $\mathrm{Cu}-\mathrm{Sb}-\mathrm{O}$ system are of considerable scientific and technological interest [1,2]. $\mathrm{CuSb}_{2} \mathrm{O}_{6}$ can be prepared by solid state reactions [3,4]. It is a $\mathrm{Cu}(\mathrm{II})$ compound for which two structural polymorphs are known [5-9]. The crystal structure of the monoclinic polymorph $\beta$ - $\mathrm{CuSb}_{2} \mathrm{O}_{6}$, space group $P 2 / n$ (14) was described by Nakua et al. [5] based on neutron powder diffraction data. This compound is reportedly stable in air up to around $1000{ }^{\circ} \mathrm{C}[9,8,7]$. The $\alpha-$ $\mathrm{CuSb}_{2} \mathrm{O}_{6}$ has a deformed trirutile-type tetragonal structure with $P 4_{2} / m n m$ (136) symmetry in which the $c$-axis of the basic rutile structure is lengthened [10]. According to Giere et al., [10] the transition from the monoclinic to the tetragonal phase occurs at ca. $106{ }^{\circ} \mathrm{C}$. There are two other known compounds that occur in the $\mathrm{Cu}-\mathrm{Sb}-\mathrm{O}$ system. $\mathrm{A} \mathrm{Cu}(\mathrm{I})$ modification with composition $\mathrm{Cu}_{8} \mathrm{Sb}_{2} \mathrm{O}_{9}$ is obtained either by thermal decomposition of $\mathrm{CuSb}_{2} \mathrm{O}_{6}$ in an oxygen-free environment, or by a solid state reaction between $\mathrm{CuSb}_{2} \mathrm{O}_{6}$ and $\mathrm{CuO}$ in air at $1120-1150{ }^{\circ} \mathrm{C}[9,8]$. A cubic $\mathrm{Cu}(\mathrm{II})-$ containing phase $\left(\mathrm{Cu}_{9} \mathrm{Sb}_{4} \mathrm{O}_{19}\right)$ also forms at $1100{ }^{\circ} \mathrm{C}$ and an oxygen pressure of 10 bar. However, on reheating in air it decomposes above $950{ }^{\circ} \mathrm{C}$ to $\mathrm{Cu}_{8} \mathrm{Sb}_{2} \mathrm{O}_{9}$ and $\mathrm{CuSb}_{2} \mathrm{O}_{6}[9,8]$.

Here we report the crystal structure of copper antimonite $\left(\mathrm{CuSb}_{2} \mathrm{O}_{4}\right)$, a compound that was previously mentioned by Mellor [11] and studied by Ricco et al. [12]. Well-crystallized material was obtained by reacting antimony trioxide with the copper-ammonium complex [13]. The SEM micrograph shown in Figure 1 reveals crystals approximately 1-5 $\mu \mathrm{m}$ in size with a pseudo-cubic appearance.

The X-ray powder diffraction data of this compound oxide could not be matched with any of the substances listed in the Powder Diffraction Files of the International Centre for Diffraction Data (ICDD) or the Inorganic Crystal Structure Database (ICSD) [14,15]. It was, therefore, decided to establish the crystal structure of this compound from the powder X-ray recordings.

The powder X-ray structural determination method used is described in detail below, followed by the computational and experimental structural verification. It is suggested here that computational 
verification of structural determinations obtained from powder X-ray data as described here should be standard practice. Although recent advances in X-ray diffraction technology have made it possible to obtain structure determinations from single crystal data for crystals as small as 8-10 $\mu \mathrm{m}$, the alternative for compounds of smaller crystal size remains a challenging task of structure solution from powder X-ray diffraction data (pXRD). A growing demand for structural information on microcrystalline materials is created by the need to characterize naturally occurring micro-minerals, but even more so by the large number of substances synthesized in powder form where growing larger crystals may not be cost effective, or too difficult to achieve. The challenges and limitations, as well as the developments and advances in the methods of structure determination from powder diffraction (SDPD) have been reviewed by numerous authors [16-20]. The determination of crystal structures from powder diffraction data remains a non-trivial process. However, the $a b$ initio SDPD method has been effectively used to successfully solve the structures of both organic and inorganic substances [20-22].

This paper presents the structure solution of copper antimonite $\left(\mathrm{CuSb}_{2} \mathrm{O}_{4}\right)$ as determined from conventional powder diffraction data, supported by supplementary information from Raman spectroscopy and the theoretical computation of the vibrational behaviour of the contents of the unit cell. Since powder X-ray structural determinations are always subject to some uncertainty, it was decided to verify the obtained structure computationally by calculating all the vibrational modes, their spectroscopic symmetries and their respective frequencies of the entire contents of the spectroscopic unit cell. The computed data were then compared with the frequencies obtained from the room-temperature Raman spectrum of the compound. Finally, results of an exploratory study of the magnetic properties are presented to contextualize the magnetic interactions against comparable copper compounds. These data are supplemented with low-temperature specific heat measurements on $\mathrm{CuSb}_{2} \mathrm{O}_{4}$. 


\section{Experimental}

Synthesis

Twinkling Star technical grade antimony trioxide (CAS-No. [1309-64-4]) powder was supplied by China MinMetals. Copper carbonate hydroxide (basic copper carbonate, CAS-No. [12069-69-1]) and ammonia solution (25\%) (CAS-No. [1336-21-6]) were obtained from Merck. The synthesis reaction was conducted in a three-neck round bottom flask fitted with magnetic stirrer and a reflux condenser. An amount of $28.25 \mathrm{~g}$ of ammonia solution (20\% stoichiometric excess) and $282 \mathrm{~g}$ of distilled water were placed in the flask and stirred at $500 \mathrm{rpm}$. To this a total of $10 \mathrm{~g} \mathrm{CuCO}_{3} \cdot \mathrm{Cu}(\mathrm{OH})_{2}$ powder was added in small portions. Following the complete dissolution of the copper carbonate hydroxide, an amount of $26.35 \mathrm{~g}$ of the antimony trioxide was added. The mixture was heated on an oil bath to reflux temperature $\left(70{ }^{\circ} \mathrm{C}\right)$. The stirrer speed was increased to $700 \mathrm{rpm}$ to maintain complete suspension of the solids. Cooling water was passed through the condenser to prevent ammonia escaping. After $6 \mathrm{~h}$ reflux, the cooling water was shut down and the ammonia was allowed to evaporate and the solid product recovered by filtration followed by drying.

\section{Characterization}

SEM imaging and EDS analysis was carried out on a Leica Stereoscan 440 SEM linked to an OXFORD INCA EDS (Energy Dispersive System). The system is equipped with an Oxford X-Max SDD detector with $20 \mathrm{~mm}^{2}$ active area and resolution of ca. $128 \mathrm{eV}$ for Mn K- $\alpha(5895 \mathrm{eV})$. EDS spot analysis was performed on polished specimens by focusing the beam on pure cuboid crystals of $\mathrm{CuSb}_{2} \mathrm{O}_{4}$ in an attempt to ensure the analytical volume is fully contained within the pure substance. Accelerating voltage was set at $20 \mathrm{kV}$, probe current at $2 \mathrm{nA}$, counting time was $100 \mathrm{~s}$ and working distance was $25 \mathrm{~mm}$. Spectra recorded from multiple measurements were quantified using the method Oxygen combined by stoichiometry and ZAF correction was applied for matrix effects. We assume the final oxide concentrations represent pure $\mathrm{CuSb}_{2} \mathrm{O}_{4}$ composition. 
High resolution imaging was obtained using an ultrahigh resolution field emission SEM (HR FEGSEM Zeiss Ultra Plus 55) with an InLens detector at acceleration voltages of $1 \mathrm{kV}$ to ensure maximum resolution of surface detail.

Raman spectra were recorded with a Horiba Jobin Yvon T64000 spectrometer, using the 514.5nm laser line of an Innova 70c coherent argon-krypton mixed gas laser as excitation source. A 50X microscope objective was used to focus the laser on the sample and the laser power was $<1$ $\mathrm{mW}$ at the sample. Spectra were collected in the range $50-1200 \mathrm{~cm}^{-1}$ with an acquisition time of $10 \mathrm{~s}$ and 2 accumulations.

The microcrystalline powder as recovered at the completion of the reaction was $1-5 \mu \mathrm{m}$ in size suitable for X-ray diffraction with no need for further micronizing. X-ray powder data were recorded on a Bruker D8 Advance diffractometer with $2.2 \mathrm{~kW} \mathrm{Cu}$ radiation $(\lambda=1.54060 \AA)$ and a LynxEye detector with $3.7^{\circ}$ active area. Samples were scanned over an angular range of $10^{\circ}$ to $132^{\circ} 2 \theta$ at a rate of $0.01^{\circ} 2 \theta$ every six seconds and generator settings of $40 \mathrm{kV}$ and $40 \mathrm{~mA}$. The powder diffraction data were collected at room temperature in the spinning mode to ensure random orientation and good particle statistics. X-ray diffractograms were also recorded after heating the material in air at $1100{ }^{\circ} \mathrm{C}$. Silicon powder $99.999 \%$ (from Sigma-Aldrich) was used as an internal standard to verify d-spacing before phase identification. Details of data collection, crystallographic and refinement data for $\mathrm{CuSb}_{2} \mathrm{O}_{4}$ are given in Table 1 .

Magnetic studies were performed on a $7 \mathrm{~T}$ field-equipped squid-type magnetometer from Quantum Design (San Diego, USA), using the high-sensitivity RSO option and $d c$ fields for the molar susceptibility data. Measurements were conducted in field-cooling mode with appropriate demagnetization between the $M$ vs. $B$ scans. Data of specific heat were collected using a heat pulse method with dual time-constant relaxation modelling on a Physical Property Measurement System from the same manufacturers with suitable calibration of the addenda present during measurement. Temperatures below $2 \mathrm{~K}$ were obtained using a helium-3 insert in the PPMS station. 


\section{Data analysis and computer modelling}

Evaluation of the diffraction data and phase identification were performed with the Bruker DIFFRACPlus-EVA Ver. 15.0.0.0 evaluation program [23]. Data analysis from the determination of space group and lattice parameters to atomic positions and structure solution was performed using the $a b$ initio methods developed by Coehlo $[17,16]$ and implemented by DIFFRAC.TOPAS Ver. 4.2 software [23]. The powder pattern for $\mathrm{CuSb}_{2} \mathrm{O}_{4}$ was indexed using the LSI algorithm based on iterative use of least squares. The process entailed peak fitting of the first 24 reflections in the angular range $10^{\circ}$ to $56^{\circ} 2 \theta$ that provided a list of peak positions and intensities for the indexing run. A $5^{\text {th }}$ order Chebychev polynomial was used for background correction and the LP factor was fixed at zero as no primary monochromator was fitted to the diffraction system. Zero error was not included at the initial fitting to allow peaks to adjust freely to the observed data. The impurity lines of the known contaminant senarmontite $\left(\mathrm{Sb}_{2} \mathrm{O}_{3}\right)$ were deselected prior to indexing. For the indexing setup, the maximum ratio of the calculated to observed reflections $\left(N_{c} / N_{o}\right)$ was set to 5 , the maximum limit for zero error was set to 0.1 and all Bravais lattices were included for testing in the indexing run. The quality of the proposed indexing solutions was determined using Chekcell [24].

The structure of $\mathrm{CuSb}_{2} \mathrm{O}_{4}$ was solved by simulated annealing (SA) in direct space. The SA algorithm is based on the effect of temperature changes on the magnitude of atomic displacements in a structural arrangement [16]. The space group and lattice parameters determined by the indexing of raw powder diffraction data of $\mathrm{CuSb}_{2} \mathrm{O}_{4}$, combined with the information on the compound stoichiometry as derived from the chemical composition and thermogravimetric analysis (TGA), were used to construct the trial crystal structure. Maximum intensity data and unconstrained individual atoms were used for the refinement. Provisions were made for the location of special positions. The input files were edited using jEdit 4.3 for TOPAS software [25]. 


\section{Computational details}

All electronic structural computations for the solid state were carried out using the MedeA suite of programs [26-28], with special reference to the programs VASP (Ver. 5.2) for crystal structure optimizations [29-31]. Standard settings were used for the VASP software parameters, while the GGA-PBEsol functional was used for the full structural optimization of all unit cell parameters (atomic positions, edge lengths and inter-edge angles), using PAW plane wave cut-off of $400 \mathrm{eV}$. The MedeA PHONON sub-suite [32] was used for phonon dispersion and phonon densities of states, as well as for the computation of the atom trajectories during the execution of the individual normal modes of vibration. The plane wave cut-off was chosen as $400 \mathrm{eV}$, while the $k$-spacing was $0.5 / \AA$, leading to a $2 \times 2 \times 2$ mesh, corresponding to an actual $k$-spacing 0 f $0.361 \times 0.361 \times 0.267 / \AA$. The symmetry analysis of the modes of vibration of contents of the spectroscopic unit cell is provided by the software, and follows the usual prescriptions of symmetry theory [33-36].

\section{Results}

Main phase identification

Powder diffractograms of the green $\mathrm{CuSb}_{2} \mathrm{O}_{4}$ synthesized at $70{ }^{\circ} \mathrm{C}$, as well as of the yellow powder obtained after heating in air to $1100{ }^{\circ} \mathrm{C}$, are shown in Figure 2. Senarmontite, $\mathrm{Sb}_{2} \mathrm{O}_{3}-F d-3 m: 2$ (No. 227) was identified in the green powder and constituted about $6.2 \mathrm{wt} . \%$ of the sample. The remaining reflections could not be matched to any of the compounds listed in the ICDD PDF-2 or ICSD. Considering the shape and spacing of the peaks it was assumed that they belong to one single phase comprising the major component in the green material. The heated sample represents a single phase product identified as $\mathrm{CuSb}_{2} \mathrm{O}_{6}$. The yellow colour is consistent with previous reports on the compound. The monoclinic structure $[3,5,6]$ showed a better match than the tetragonal $[10]$ and is thus consistent with the $\beta$ - $\mathrm{CuSb}_{2} \mathrm{O}_{6}$ polymorph. This was confirmed by Chekcell with $P 2{ }_{1} / n$ 
symmetry as the best cell solution. The heated product was of high purity suggesting that the senarmontite impurities might have evaporated during the heat treatment step.

\section{Composition}

The chemical formula for the synthesized copper antimonite product, as calculated from EDS analysis, was $\mathrm{Cu}_{0.95} \mathrm{Sb}_{2.03} \mathrm{O}_{4}$. TGA analysis showed a mass increase of $7.8 \mathrm{wt} \%$ upon heating to $1000{ }^{\circ} \mathrm{C}$ [13]. The theoretical expected mass increase for the oxidation of $\mathrm{CuSb}_{2} \mathrm{O}_{4}$ to $\mathrm{CuSb}_{2} \mathrm{O}_{6}$ is 8.6 wt. \%. The minor discrepancies between these empirical observations and the theoretical expectations are attributed to the presence of the minor amount of antimony trioxide impurity.

\section{Unit cell determination}

The powder pattern of the green antimony oxide was indexed on a tetragonal unit cell $P 4_{2} b c(106)$ with the highest goodness of fit (GOF). All 24 selected lines were successfully indexed, together with four additional theoretical lines, i.e. lines of low intensities were added to the list in the process. A calculated zero error of 0.0743 and impurity lines removal corrections were successfully applied with reproducible figures of merit at low $R_{\mathrm{wp}}$ values. Multiple Pawley refinement of the top 67 solutions produced a list of suggestions with best $R_{\mathrm{wp}}=0.0664$ for space group $P 4_{2} b c$ and $R_{\mathrm{wp}}$ of 0.0673 for cell $P 4_{21} c$. Both cells are of tetragonal symmetry and almost identical volumes and cell parameters. Evaluation of the top ten $R_{\mathrm{wp}}$ solutions by Chekcell confirmed $\mathrm{P} 4_{2} b c$ as the best space group for the raw data with refined cell parameters $a=b=8.76033(5) \AA, c=5.79786(4) \AA$ and $V=$ $444.947(5) \AA^{3}$. It denotes a primitive centring with a fourfold screw axis and glide translations along half $b$ and $c$ axis. Crystallographic and refinement data for $\mathrm{CuSb}_{2} \mathrm{O}_{4}$, together with details of data collection and instrument conditions are shown in Table 1.

Structure solution 
Based on the experimental confirmation of $\mathrm{CuSb}_{2} \mathrm{O}_{4}$ stoichiometry the number of formula units $(Z)$ in the unit cell was calculated to be 4 . With this information in hand the trial crystal structure was constructed with 7 unconstrained individual atoms on arbitrary general positions. Provision for identification of special positions was made as well as calculation of fractional coordinates and bond lengths and angles. Non-structural parameters, i.e. instrument parameters, background, Zero error, LP correction, etc. were determined by Pawley refinement of the raw data for $P 4_{2} b c$ cell and served as basis input file for structure solution. Identical occupancies for the $\mathrm{Sb}$ and $\mathrm{O}$ atoms were detected after the first cycle, causing clustering of atoms during refinement. Similarly $\mathrm{Cu}$ atoms were sitting on top of each other with close to zero bond length and half site occupation. The degenerate $\mathrm{Sb}$ and $\mathrm{O}$ atoms located on same sites with equivalent positions were removed from the structure whereas special position values for $x, y$ and $z$ coordinates of the $\mathrm{Cu}$ atom were appropriately assigned. Furthermore, the $\mathrm{Cu}$ and $\mathrm{Sb}$ atoms were constrained but still allowed to adjust positions within defined limits of $0.25 \AA$. The special positions and the bond length and angles were fixed for further refinements. The structure was considered solved at $R_{\mathrm{wp}}=0.0630$. The atomic coordinates and occupancies are listed in Table $2 . \mathrm{Sb}^{3+}$ and both $\mathrm{O}^{2-}$ atoms occupy general positions with Wyckoff sites of $8 \mathrm{c}$ as per $\mathrm{P}_{2} b c$ symmetry. Because of its special positions the $\mathrm{Cu}^{2+}$ atom was assigned smaller multiplicity of $4 b$. There are 28 atoms within the 4 formula units in the unit cell. A subsequent refinement converged to $R_{\mathrm{wp}}=0.0398$ with R-Bragg value of 0.0182 . The crystal structure of $\mathrm{CuSb}_{2} \mathrm{O}_{4}$ is presented in Figure 3. Powder X-ray diffraction data for tetragonal $\mathrm{CuSb}_{2} \mathrm{O}_{4}$ in the angular range $10-132^{\circ} 2 \theta$ is presented in Table S.1 of the Supplementary Material.

\section{Description of the crystal structure}

The structure is build-up of chains of edge-sharing $\mathrm{CuO}_{6}$ octahedra extending along the $\mathrm{c}$ axis. These chains are laterally linked by pyramidal $\mathrm{SbO}_{3}$ polyhedra that also link to each other in a direction parallel to the $\mathrm{CuO}_{6}$ chains. Under $\mathrm{P}_{2} b c$ symmetry these form a somewhat twisted framework. The 
octahedrally coordinated $\mathrm{Cu}^{2+}$ forms axially elongated $\mathrm{Cu}$ polyhedra. This structural distortion is typically attributed to the Jahn-Teller type coordination around $\mathrm{Cu}^{2+}$ ions, which is usually manifested by four (or two) short and two (or four) long $\mathrm{Cu}-\mathrm{O}$ bonds. Such an atomic arrangement results in either elongated [4+2] or compressed [2+4] octahedron [37]. This, however, is not exactly the case in the $\mathrm{CuSb}_{2} \mathrm{O}_{4}$ structure determined in the present study. The $\mathrm{CuO}_{6}$ polyhedron has two long bonds, two intermediate and two short bonds (see Figure 4). The two axial $\mathrm{Cu}-\mathrm{O} 1$ long bonds are 2.447(13) A with an inter-bond angle O1-Cu-O1 of $172.28(10)^{\circ}$. The four equatorial Cu-O2 bonds display a more complex geometrical arrangement (see Figure 4 and Table S.2 of the Supplementary Material). Two neighbouring $\mathrm{Cu}-\mathrm{O} 2$ bonds of intermediate distance 2.07(3) $\AA$ are formed with an inter-bond angle of $80.55(12)^{\circ}$, while the short distance pair $\mathrm{Cu}-\mathrm{O} 2$ has a length of $1.88(2) \AA$ and an inter-bond angle of $90.85(16)^{\circ}$. Such a structural pattern has been described as $[2+2+2]$ coordination in $\mathrm{Cu}^{2+}$ oxysalt minerals $[38,39]$. The moiety contains a central rhombic "chair-like" unit formed by the non-bonded $\mathrm{Cu}-\mathrm{Cu}$ atoms with distance $2.899 \AA$ and O2-O2 at the opposite diagonal of $2.677 \AA$. The $\mathrm{SbO}_{3}$ pyramidal configuration is almost regular with $\mathrm{Sb}-\mathrm{O} 1$ bonds of 1.97(2) $\AA(2 \mathrm{x})$ and Sb-O2 of 1.959(5) $\AA$. The inter-bond angles O1-Sb-O1 are $95.50(4)^{\circ}, \mathrm{O} 1-\mathrm{Sb}-$ $\mathrm{O} 2-93.80(10)^{\circ}$ and $91.50(8)^{\circ}$ for O2-Sb-O1, respectively (see Figure 5 and Table S.2 of the Supplementary Material).

Verification by computer modelling studies

The full VASP/PBEsol electronic optimization changed the cell parameters $a, b$, and $c$ by about $-0.8 \%,-0.8 \%$ and $+1.3 \%$, respectively, while it left the angles $\alpha, \beta, \gamma$ unchanged. The fractional coordinates of the atoms in the unit cell changed by \pm 0.002 during the full optimization. The full optimization of all unit cell parameters $(a, b, c$ and angles $\alpha, \beta, \gamma)$ proceeded smoothly and showed that the experimental determination of the space group and unit cell and its contents are essentially correct. 
The PHONON computation of the green compound, $\mathrm{CuSb}_{2} \mathrm{O}_{4}$, which crystallizes in the tetragonal system with space group $P 4_{2} b c-C_{4 v}{ }^{8}$ (106), with $Z=4$, was done with the geometry of the VASP-optimized unit cell described above. There are thus $(3 N=84)$ vibrational modes in the unit cell generated by the harmonic motions of its $N=28$ atoms, since the spectroscopic unit cell is equal to the actual unit cell in the case of this space group. The following irreducible representations under the point group $C_{4 v}$ are found for the vibrations of this spectroscopic unit cell, namely $\Gamma=10 A_{1}+10 A_{2}+10 B_{1}+10 B_{2}+22 E$

A symmetry analysis shows that the vibrational modes belonging to the $A_{1}$ representation are both Raman and IR active, while those belonging to the $A_{2}$ representation are inactive in both the Raman, as well as in the infrared. The vibrational modes belonging to the $B_{1}$ and $B_{2}$ vibrations are only Raman active, while the doubly-degenerate $E$ modes are active in both the Raman and the IR. This means that maximally 30 lines in the Raman spectrum of the solid are due to the scattering of 30 singly-degenerate vibrational modes, while 22 lines are due to the doubly-degenerate E modes, that is, there are maximally 52 lines which may appear in the Raman spectrum if all the Ramanscattering modes are resolved by the instrumentation. Ten vibrational modes of the unit cell are inactive in the Raman and in the infrared. The PHONON computation yielded all 84 possible modes, their symmetry assignments, spectroscopic activities and respective vibrational frequencies as displayed in Table 3, which also lists the 10 spectroscopically inactive $A_{2}$ modes and their frequencies. Six of these 84 modes with the lowest frequencies are not true internal vibrational modes of the contents of the unit cell.

The MedeA/PHONON computation unfortunately only provides the vibrational frequencies and their respective symmetries and not a "picture" of the actual Raman and IR spectral traces that can be expected. However, it is usually possible to compare the computed vibrational frequencies with the experimentally-measured Raman frequencies, keeping the spectroscopic activity in mind. 
Table 3 lists the PHONON computed unit cell vibrational modes and the corresponding modes identified in the experimental Raman spectrum, which is displayed in Figure 6. Many of the observed Raman bands in Figure 6 can be considered to be fairly broad when compared with the close proximity of some of the computed frequencies. Such a broad band in the experimental spectrum can be interpreted as an envelope covering the Raman scattering of more than one closely adjacent vibrational mode. This effect explains the paucity of experimentally-found frequencies compared with the computed number of frequencies.

It is, furthermore, presumed that the optically active vibrational modes, which do not appear in the Raman spectrum, do not have appreciable values for $\partial \alpha_{i} / \partial Q_{i}$. The Raman peaks may probably be sharpened by cooling the sample down to $4 \mathrm{~K}$, at the same time resolving some of the closely separated peaks and/or allowing the low-intensity peaks to be observed.

The bands, due to the $\mathrm{Sb}_{2} \mathrm{O}_{3}$ contamination, which occur at $84.9,191.1,235.3$ and $449.0 \mathrm{~cm}^{-1}$ in the spectrum of the sample, are not shown in Table 3 and in Figure 6. It should be pointed out that the shoulders at 231 and $235 \mathrm{~cm}^{-1}$ in Figure 6 are probably artefacts due to the presence of $\mathrm{Sb}_{2} \mathrm{O}_{3}$. The wavering trace between about $40 \mathrm{~cm}^{-1}$ and up to the shoulder of the large peak at $110 \mathrm{~cm}^{-1}$, may either be due to some background noise, or may actually represent some of the modes $8,9,11$ to 15 .

It is to be noted that mode 36 , which appears as a strong band at $322 \mathrm{~cm}^{-1}$, actually represents the nodes that are accidentally degenerate (B2 and E), thus bringing the total number of modes up to the required 62 . The very weak band at $589 \mathrm{~cm}^{-1}$ may represent any (or all) of the nodes 52 to 54 . The broad band at $790 \mathrm{~cm}^{-1}$ may represent modes 59 and/or mode 60 .

There are five small imaginary frequencies found in the vibrational computation of the unoptimized unit cell, which are reduced to the four (smaller) values shown in Table 3 after the full optimization of the unit cell (cell dimensions, cell angles and fractional coordinates). They are probably caused by a combination of some numerical effects, by the failure of the DFT-functional to 
properly apply the Eckhard-Sayvetz conditions [33], as well as by the presence of the $\mathrm{Sb}_{2} \mathrm{O}_{3}$ impurity in the form of either twinning or the formation of a limited range of mixed crystals.

The presence of such small negative or positive frequencies amongst the six lower frequencies of any molecular optimization, followed by a frequency computation is common amongst most, if not all, such computational programs. This happens even in the case of an isolated molecule, such as water, when optimized using a DFT/B3LYP/6-311++G(3df,3dp) methodology, where the six lowest of the $3 N$ frequencies are found to be $-78.1424,-67.2020,-55.4972,0.0015,0.0016$, and 0.0017 $\mathrm{cm}^{-1}$.

If good Raman and IR spectra are available for a well-defined pure compound for which a crystal structure determination from a single crystal (no twinning) is also available, it is usually a good idea to compute the normal modes of vibration of the contents of the spectroscopic unit cell. This allows to substantiate the correctness of the synthesis of the compound (purity, the quantity of contaminants), as well as verify the correctness and accuracy of the crystal structure determination and to assist in the assignment of the observed vibrational bands. The crystal structure (provided a reasonable $R$-factor is obtained) is used as input for the vibrational computation, namely: (i) the space group, (ii) the number of atoms in the unit cell, (iii) the fractional coordinates $(x, y, z)$ for each atom, (iv) the assurance that there are no fractional occupancies for every atom position.

The computational program for the vibrational modes assumes that all atoms in the unit cell are on positions of potential-energy equilibrium, but if the computational program has the option builtin, it can also optimize both the unit cell $(a, b, c, \alpha, \beta, \gamma)$ and all the atomic positions before a vibrational calculation is executed. A crystal structure determination with errors in one or more of (i), (ii), (iii) or (iv) will either not compute or will give imaginary (large negative, sometimes up to $-1500 \mathrm{~cm}^{-1}$ ) frequencies for one or more vibrational modes when the atoms involved in their execution are not in position of equilibrium or if the space group is wrong. If the optimization 
process does not reduce the imaginary frequencies markedly, then the crystal structure determination is almost certainly wrong.

However, (small) imaginary computed frequencies can also be due to a number of factors that act separately, but are summed together at the end. These are (a) too few decimals in the cell parameters $(a, b, c, \alpha, \beta, \gamma)$, (b) too few decimals in the fractional atomic coordinates, (c) (large) errors in the cell parameters, (d) (large) errors in one or more fractional coordinates, (e) rounding-off and other numeric processes in the computational program (e.g. in diagonalization sub-routines), (f) the use of a basis function that is not quite optimized for all the types of atoms in the unit cell (especially for the heavier atoms), (g) if the crystal contains some small amounts of impurities embedded in the crystal itself (e.g. twinning, solid solution formation, such as $\mathrm{Sb}_{2} \mathrm{O}_{3}$ in this case), the structure may be slightly distorted from the equilibrium structure, leading to (small) negative frequencies, and (h) non-fulfilment of the Eckard-Sayvetz conditions [33] that ensure the separation of the translational and rotational modes of the unit cell from the internal modes of the cell [33], which may inter alia be due to the unsuitability of the chosen basis function or the functional for the atoms in the unit cell. Errors (a) to (d) should ideally be corrected by an optimization process, but errors (e) to (h) cannot be corrected in this way. If one or more smaller negative frequencies still occur in the computed vibrational spectrum, then factors (e to h) may play a role. The question is: if an optimization makes the imaginary frequencies less negative, just where is the dividing line between a correct structure and an incorrect one? It is found by trial and error that an incorrect crystal structure (incorrect space group, cell parameters and/or fractional coordinates) give rise to (negative) imaginary frequencies in the range of $-70 \mathrm{~cm}^{-1}$ to several thousand negative wave numbers. Less than about $-70 \mathrm{~cm}^{-1}$ may be regarded as being due to errors (e) to (h) above.

The arguments above can be summarized by the following statement: IF the theoretically computed unit cell vibrational modes agree with experimentally observed frequencies (up to the experimental error bounds) AND if there are no large imaginary frequencies amongst the set of $3 \mathrm{~N}$ 
computed frequencies (where $\mathrm{N}$ is the number of atoms in the unit cell) which cannot be further improved by optimization, THEN it can be concluded that the crystal structure as determined, is correct. Since the crystal structure of $\mathrm{CuSb}_{2} \mathrm{O}_{4}$ was determined from X-ray powder diffraction data as refined by the Rietveld method, it was subjected for verification to a detailed computational study. The vibrational computation using the experimental unit cell determined in this study, showed the presence of some modes (Table 3) with small negative frequencies, but which fall in the range that can be accepted since the sample contained some $\mathrm{Sb}_{2} \mathrm{O}_{3}$ as distorting impurity. In addition, the experimental Raman frequencies agree with the computed modes within a few $\mathrm{cm}^{-1}$ (see Table 3 ). Unfortunately, the IR spectrum shows too many large and very broad bands to contribute appreciably to the assignment.

\section{Comparison to other similar structures}

A significant number of $\mathrm{Cu}^{2+}$ structural varieties have been reported to date, which most often are not isostructural with non- $\mathrm{Cu}^{2+}$ analogues $[5,9,8]$. To a large extent this is due to the diversity of distorted coordination geometry in the $\mathrm{Cu}^{2+}$ polyhedron associated with the well-known Jahn-Teller effect. Compounds of general formula $\mathrm{MSb}_{2} \mathrm{O}_{4}(\mathrm{M}=\mathrm{Mg}, \mathrm{Mn}, \mathrm{Fe}, \mathrm{Ni}, \mathrm{Zn})$ are isostructural in tetragonal symmetry with $\mathrm{M}^{2+}$ elongated polyhedra in [4+2] coordination [40,41]. In the case where $\mathrm{M}=\mathrm{Cu}^{2+}$ as in $\mathrm{CuSb}_{2} \mathrm{O}_{4}$ the structure has to adjust to accommodate the distortion introduced by the Jahn-Teller effect on the $\mathrm{Cu}^{2+}$ octahedron. This structural adjustment results in a $[2+2+2]$ configuration that appear energetically more favourable for $\mathrm{Cu}^{2+}$ sites in the structure. Similar atomic arrangement has been reported to be a relatively common coordination in $\mathrm{Cu}^{2+}$ oxysalt minerals [39]. In the $\mathrm{CuSb}_{2} \mathrm{O}_{6}$ compound the configuration of $\mathrm{Cu}^{2+}$ varies from compressed [2+4] polyhedral type in the tetragonal $\left(\mathrm{P} 4_{2} / \mathrm{mnm}\right)$ structure to elongated $[2+2+2]$ in the monoclinic $\left(\mathrm{P} 2_{1} / \mathrm{n}\right)$.

\section{Magnetic studies}


In view of a more than superficial similarity in the compound formulae between the title compound $\mathrm{CuSb}_{2} \mathrm{O}_{4}$ and that of the well-known higher oxide $\mathrm{CuSb}_{2} \mathrm{O}_{6}$, it is of interest to compare crystallographic as well as physical properties between these compounds in order to investigate how the different atomic environments impact upon their respective properties. Below, we briefly summarize aspects of the properties of $\mathrm{CuSb}_{2} \mathrm{O}_{6}$ pertinent to our study of $\mathrm{CuSb}_{2} \mathrm{O}_{4}$.

In the monoclinically distorted trirutile type structure [5] $\mathrm{CuSb}_{2} \mathrm{O}_{6}$ has nearest-neighbour $\mathrm{Cu}^{2+}$ ions separated by $4.6349 \AA$, and next-nearest neighbours only slightly further apart at $4.6370 \AA$. In the present compound $\left(\mathrm{CuSb}_{2} \mathrm{O}_{4}\right)$ the corresponding distances are $2.899 \AA$ and $6.194 \AA$ respectively.

Above $\sim 20 \mathrm{~K}$ small but distinct magneto-crystalline anisotropy is found in $\mathrm{CuSb}_{2} \mathrm{O}_{6}$ [42] which is reflected in the similarity among the Landé values (from $g=2.18$ along the $a$-axis to $g=2.26$ along $c$ ), and which means that the effective moment acting among the three directions is in fact rather isotropic. However, a large disparity is found between the interchain to intrachain exchange interaction values $\left(\mathrm{J}_{\text {inter }} / \mathrm{J}_{\text {intra }}=3 \%\right)$ in $\mathrm{CuSb}_{2} \mathrm{O}_{6}$. The controversies regarding the way in which properties are influenced by details of the crystal environment in $\mathrm{CuSb}_{2} \mathrm{O}_{6}$ appear to be unresolved. The lattice is amenable to a 2-dimensional description whereas clear 1-dimensional behaviour is found in the magnetic behaviour (see Kasinathan et al. [2], and references therein). Recent work expanding the suite of physical data on $\mathrm{CuSb}_{2} \mathrm{O}_{6}$ to include thermal expansion as well [43], gave evidence of a 3-dimensional gap governing the heat capacity below $T_{\mathrm{N}}$, with distinct jumps in the thermal conductivity at $T_{\mathrm{N}}$. Hence, a novel description was forwarded in terms of 1-dimensional $S=$ $1 / 2$ ordering being responsible for the broad peak in susceptibility at $115 \mathrm{~K}$, and which is followed at 8.6 K by antiferromagnetic ordering of a 3-dimensional nature. At the same time, the large value of $J_{\text {intra }}$ compared to $J_{\text {inter }}[42]$ means that the view of a 1-dimensional nature in $\mathrm{CuSb}_{2} \mathrm{O}_{6}$, at least as far as the spin arrangement is concerned, is notionally correct. The electronic structure study of Kasinathan et al.[2] gave credence to the chains in $\mathrm{CuSb}_{2} \mathrm{O}_{6}$ being nominally along the [110] direction. An elastic neutron scattering study [44] modelled the antiferromagnetic order among $\mathrm{Cu}^{2+}$ 
ions by means of an ordered moment of $0.51 \mu_{\mathrm{B}}$, arranged within the $a-b$ plane, and with a dominant component along the $b$-axis. This picture is in good accord with the magnetic susceptibility which showed that the decrease in susceptibility below $T_{\mathrm{N}}$ is most pronounced when gauged along the $a$ axis.

Our data of the $B=0.1$ T fixed-field magnetic susceptibility $\chi(T)$ of $\mathrm{CuSb}_{2} \mathrm{O}_{4}$ is shown in Fig. 7 . $\chi(T)$ is strongly temperature dependent, which points to the involvement of $\mathrm{Cu}^{2+}$ as a magnetically active species in this compound. The magnitude of $\chi(T)$ towards low temperature exceeds that of $\mathrm{CuSb}_{2} \mathrm{O}_{6}[42]$ by a factor of $\sim 100$. This may qualitatively be attributed to the much shorter nearestneighbour and non-bonding distances found in $\mathrm{CuSb}_{2} \mathrm{O}_{4}$ compared to that of $\mathrm{CuSb}_{2} \mathrm{O}_{6}$ (see parameters cited further above). It is noted that there is no Curie-like component detected that may arise from a spurious magnetic phase or phase mixture of the title compound or otherwise from, for example, aggregation of a magnetic species in grain boundaries. Furthermore, the higher-temperature susceptibility is found not to follow a Curie-Weiss law. However, an unambiguous interpretation of this point should await the availability of single-crystal material in order to clarify the possibility that magneto-crystalline anisotropy, the presence of which in $\mathrm{CuSb}_{2} \mathrm{O}_{4}$ is supported by calculations described further below, may in fact be responsible for accidentally concealing Curie-Weiss behaviour.

The overall shape of $\chi(T)$ illustrated in Figure 7 and its inclination towards a broad peak at intermediate temperatures is consistent with the susceptibility expected for an arrangement of magnetic one-dimensional (1D) Heisenberg chains. This situation is well suited to the case of halfinteger spin, $S=1 / 2 \mathrm{Cu}^{2+}$ ions in $\mathrm{CuSb}_{2} \mathrm{O}_{4}$. The attraction of experiments on spin systems of this nature lies in the uniqueness of exact solutions worked out by Yang and Yang [45-47]. The broad peak is attributed to magnetic exchange that precipitates into weak, short-range order. We refer to the work of Hatfield [48] in an attempt at understanding the magnetism of $\mathrm{CuSb}_{2} \mathrm{O}_{4}$. Analytical relations were proposed by Hatfield [48] which enable a comparison to be drawn between interchain and 
intrachain exchange interactions. The solid line superimposed onto our data in the main panel of Figure 7 represents a best fit to the Hatfield expressions for intrachain isotropic exchange coupled $S$ $=1 / 2$ spins. From a summative assessment of the $\mathrm{Cu}^{2+}, \mathrm{Sb}^{5+}$, and $\mathrm{O}^{2+}$ elemental diamagnetic contributions $\left(\approx 87 \times 10^{-6} \mathrm{~cm}^{3} / \mathrm{mol}\right)$, the need to correct for a diamagnetic component in our fit procedure is obviated. Aside from coefficients scaling the magnetic exchange to thermal energy factors that are involved $\left(J / \mathrm{k}_{\mathrm{B}} T\right)$, the important parameter which is obtained through the fit is $|J| / \mathrm{k}_{\mathrm{B}}=$ 5.40(5). The fit that constitutes this parameter reproduces the main features shown in Figure 7 in a near-quantitative manner. However, as is discussed further below, we have in addition to the putative short-range order in $\mathrm{CuSb}_{2} \mathrm{O}_{4}$ also uncovered a much more robust and long-range magnetic order at low temperature $\left(T_{\mathrm{m}}=1.8 \mathrm{~K}\right)$, which means that the spins in this compound are condensed in its ground state and for this we need to examine forms of magnetic exchange beyond nearest-neighbour exchange. We have applied the expression developed by Hatfield pertinent for incorporating interchain magnetic exchange $J_{\text {inter }}$, in the presence of the above $|J|$ between atoms adjacent to each other within a chain. This produces a fit representing the data very accurately (not shown) and parametrized by $|J|_{\text {inter }} / \mathrm{k}_{\mathrm{B}}=0.010(5)$, which is of the order 100 times weaker that the intrachain exchange. This ratio argues in terms of chains that are considered effectively as isolated in $\mathrm{CuSb}_{2} \mathrm{O}_{4}$, as was similarly found in terms of the value of $\approx 3 \%$ [42] obtained for $J / J_{\text {intra }}$ in $\mathrm{CuSb}_{2} \mathrm{O}_{6}$. By further way of comparison, analysis of the susceptibility in a single crystal of $\mathrm{CuSb}_{2} \mathrm{O}_{6}$ [42] led to Landé factor values that differed by less than $4 \%$ among the three crystal directions, and consequently only very small magnetocrystalline anisotropy in $\chi(T)$ among the 3 major crystal axes. For the present case of $\mathrm{CuSb}_{2} \mathrm{O}_{4}$ our analysis on polycrystalline sample material yields $g / g_{\text {intra }} \approx 2.4$, and we anticipate therefore that single crystals are likely to reveal considerable anisotropy in the magnetic properties of this compound. The magnetization $M(B)$ of $\mathrm{CuSb}_{2} \mathrm{O}_{4}$ measured at $T=1.76 \mathrm{~K}$ (inset, Figure 7) produces near-linear behaviour which conveys a field-independent susceptibility up to $B$ $\approx 5 \mathrm{~T}$. A weak upturn observed beyond this field is likely due to metamagnetism, but considerably 
higher fields will be needed to evaluate this conjecture. Inspection of the $M(B)$ data at $1.76 \mathrm{~K}$ in very low fields showed no ferromagnetic component to within the levels of detection. In the following, we turn to the low-temperature susceptibility results on $\mathrm{CuSb}_{2} \mathrm{O}_{4}$.

$\chi(T)$ at low temperature and in different applied magnetic fields reveal detail that deserves further scrutiny. The low-field region of the magnetization (inset, Fig. 7) tracts a near linear-in-field behaviour, but high-resolution susceptibility measurements enabled resolution of a weak field dependence of $c(T)$, as can be seen from the data in Fig. 8 collected in fields up to 1T. The features associated with low-dimensional magnetism are retained nevertheless and we confirm, as shown in Figure 8, that the maximum found in $\chi(T)$ shifts upward in increasing field, which supports the interpretation of this feature as a collective, magnetic effect in $\mathrm{CuSb}_{2} \mathrm{O}_{4}$. The temperature location of the susceptibility maximum for an isotropic Heisenberg chain is predicted as $\mathrm{k}_{\mathrm{B}} T_{\max } / \mathrm{J} \mid=1.282$ [49], which agrees to a fair measure with the value $\approx 1$ for this ratio in our data.

At the low-temperature limit of $\chi(T)$, a well-defined break in the slope is found below about $T_{\mathrm{m}}$ $=1.8 \mathrm{~K}$. We attribute this feature to the onset of long-range magnetic order, probably of antiferromagnetic nature on account of the absence of a ferromagnetic signal in the magnetization $M(B)$ (Fig. 7). The anomaly in $\chi(T)$ at $T_{\mathrm{m}}$ weakens progressively in higher applied fields. In the comparable compound $\mathrm{CuSb}_{2} \mathrm{O}_{6}$ similar ordering occurs below $8.5 \mathrm{~K}$ [5]. Thus, the two ordering temperatures in this and the title compound scale qualitatively according to their respective exchange interaction parameters.

The specific heat in the form $C_{\mathrm{p}}(T) / T$ of $\mathrm{CuSb}_{2} \mathrm{O}_{4}$ is illustrated in Figure 9(a). The phase transition at $T_{\mathrm{m}}=1.8 \mathrm{~K}$ discussed above is confirmed in $C_{\mathrm{p}}(T) / T$, with the transition temperature taken as is customary at the temperature of maximum slope on the high-temperature side of the transition (green arrow). The specific heat reaches a peak of $C_{\mathrm{p}}(\mathrm{T}) \approx 1.36 \mathrm{~J} / \mathrm{mol}$.K just below $T_{\mathrm{m}}$. In the inset to Figure 9(a), we have cast the specific heat against $T^{2}$, a form which is amenable to evaluating the electronic specific heat. This is a form that rigorously lends itself to the free-electron 
model of metals, but we note that the same temperature dependence was calculated also for the TA0 limiting specific heat of a $S=1 / 2$ isotropic Heisenberg chain [50]. For $\mathrm{CuSb}_{2} \mathrm{O}_{4}$, we found $C_{\mathrm{p}}(T A 0) / T \approx 0.577 \mathrm{~J} / \mathrm{mol} . \mathrm{K}^{2}$. This value is approximately half of what is predicted by Johnston et al. [50], i.e., $C_{\mathrm{p}}(T A 0) / T=2 R / 3|J|=1.026 \mathrm{~J} / \mathrm{mol} . \mathrm{K}^{2}$ in terms of $|J|=5.4$ obtained from our susceptibility analysis. The reason for this shortfall is not clear at first sight. However, inspection of the entropy plotted in Figure 9(b) provides some clues on this observation, in which the total entropy across the phase transition at $T_{\mathrm{m}}$ was calculated according to $S=\int_{0}^{T^{\prime}} C_{p}(T) / T \cdot d T$. At a magnetic phase transition involving all of the magnetic species, an $S=1 / 2$ spin system should liberate an amount $S=$ $R \ln (2)$ of configurational entropy. Figure 9(b) shows that no more than $\sim 10 \%$ of this amount of entropy is being occupied at $T_{\mathrm{m}}$, but there is a considerable rise in entropy beyond the phase transition. This is probably due to the major part of the spin entropy being tied up in short-range correlations associated at least partly with the peak feature in susceptibility at $\approx 5.5 \mathrm{~K}$.

Finally, we note in the specific heat shown in Figure 9 a further feature well within the magnetically ordered region, having an apex close to $0.9 \mathrm{~K}$. The nature of this feature is unknown at present, but the well-defined anomaly that it traces in $C_{\mathrm{p}}(T) / T$ supports the notion that this is a further bulk, cooperative ordering phenomenon near the ground state of $\mathrm{CuSb}_{2} \mathrm{O}_{4}$. Elucidation of this feature requires further low-temperature measurements. As a consequence of the additional structure that this feature produces in the specific heat, an exact assessment of the low-temperature specific heat attributable to $T_{\mathrm{m}}$ is also not possible without being able to deconvolute their respective contributions. For this purpose it would be beneficial to study the specific heat in applied magnetic fields, together with a collection of other properties such as magnetic susceptibility below the temperatures presented in this survey.

\section{Conclusions}


Copper antimonite $\left(\mathrm{CuSb}_{2} \mathrm{O}_{4}\right)$ was successfully synthesized via the reaction of antimony trioxide powder with aqueous copper-ammonia complex at reflux conditions. A well-crystallized product with a cuboid crystal habit approximately $1-5 \mu \mathrm{m}$ in size was obtained. Its crystal structure was determined from the X-ray powder diffraction data. Simulated annealing (SA) in direct space using the Rietveld method indicated tetragonal symmetry, space group $P 4_{2} b c$ (106), with unit cell

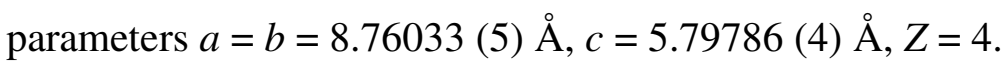

Computational verification of this experimentally-determined structure proceeded through two stages, namely, (i) the optimization of the atomic positions, edge lengths and inter-edge angles of the unit cell with respect to the total electronic energy of its contents, using the program VASP 5.2 of the MedeA suite of computational programs with standard setting and the PBEsol functional., and (ii) since a vibrational analysis of any molecular structure can only be carried out when the atomic system finds itself on a potential minimum, this optimized unit cell was used as input for the PHONON calculation.

The correspondence between the computed and experimentally determined Raman frequencies support the assumption that the crystal structure is correctly determined as reported here. The same conclusion follows from the full optimization, which does not for all intents and purposes change any of the reported crystallographic data (space group, cell dimensions and fractional atomic coordinates).

The magnetic susceptibility and specific heat data obtained for $\mathrm{CuSb}_{2} \mathrm{O}_{4}$ indicate that this compound is an interesting new addition to the cohort of $\mathrm{Cu}^{2+}$ compounds known to exhibit lowdimensionality and with remarkable consequences for their ground states and cooperative behaviour. The fascination of low-dimensional $\mathrm{Cu}$ systems has been expressed most widely in the 2D superconducting cuprates. At the same time, the procurement of $S=1 / 2$ ladder systems and related low-dimensional $\mathrm{Cu}$ geometries in the middle 80's have spurned renewed interest in what had already been known at the time as exact theoretical solutions for certain $S=1 / 2$ case studies. We 
have provided compelling evidence for the title compound $\mathrm{CuSb}_{2} \mathrm{O}_{4}$ belonging to the class of $S=1 / 2$ Heisenberg chain systems. The magnetic susceptibility in particular signifies a spin system of this nature and its coupling to an applied magnetic field, but in our specific heat results clear evidence was found for the additional ordering which takes place among $\mathrm{Cu}^{2+}$ ions below $T_{\mathrm{m}}=1.8 \mathrm{~K}$ as well in this compound.

Supporting Information: Crystallographic data in CIF format. X-ray Powder Diffraction Data for $\mathrm{CuSb}_{2} \mathrm{O}_{4}$ (Table S.1). Geometric Parameters for $\mathrm{CuSb}_{2} \mathrm{O}_{4}$ (Table S.2). Experimental Raman spectra (Figure S.1). Photograph of the $\mathrm{CuSb}_{2} \mathrm{O}_{4}$ and $\mathrm{CuSb}_{2} \mathrm{O}_{6}$ powders (Figure S.2). TGA-DTA curve for $\mathrm{CuSb}_{2} \mathrm{O}_{4}$ heated in air (Figure S.3). Rietveld quantitative results for $\mathrm{CuSb}_{2} \mathrm{O}_{4}$ and $\mathrm{Sb}_{2} \mathrm{O}_{3}$ (Figure S.4).

\section{Acknowledgments}

Acknowledgments Financial support for this research from AEL Mining Services and the THRIP program of the Department of Trade and Industry and the National Research Foundation of South Africa is gratefully acknowledged. AMS thanks the NRF (78832) and the URC of UJ for financial assistance.

\section{References}

1. Stan M, Mihaiu S, Crisan D, Zaharescu M (1998) Subsolidus phase equilibrium in the Cu-Sb-O system. European Journal of Solid State and Inorganic Chemistry 35 (3):243-254

2. Kasinathan D, Koepernik K, Rosner H (2008) Quasi-one-dimensional magnetism driven by unusual orbital ordering in CuSb2O6. Physical Review Letters 100 (23)

3. Swanson HEM, H.F.; Morris, M.C.; Evans, E.H. (1967) Standard X-ray Diffraction Powder Patterns, vol Section 5. NBS Monograph 25. National Bureau of Standards, Washington DC

4. Simada S, Mackenzie KJD (1982) Solid-state reactions in the system Cu-Sb-O; Formation of a new copper(I) antimony oxide. Thermochimica Acta 56 (1):73-82

5. Nakua A, Yun H, Reimers JN, Greedan JE, Stager CV (1991) Crystal structure, short range and long range magnetic ordering in CuSb2O6. Journal of Solid State Chemistry 91 (1):105-112

6. Ramos El, J.; Galtan, M.; Veiga, M.L. (1991) Synthesis and structural characterization of Sb(V) oxides with rutiie and trirutile structures. Anales de Química 87:966-969

7. Byström AH, B.; Mason, B. (1941) The crystal structure of zinc metantimonate and similar compounds. Arkiv for Kemi, Mineralogi och Geologi 15B (4):1-8 
8. Shimada S, Kodaira K, Matsushita T (1985) Preparation and characterization of a new copper antimony oxide, Cu9Sb4O19. Journal of Solid State Chemistry 59 (2):237-241. doi:http://dx.doi.org/10.1016/00224596(85)90323-8

9. Shimada S, Mackenzie KJD, Kodaira K, Matsushita T, Ishii T (1988) Formation of new copper antimony oxides by solid state reaction between $\mathrm{CuSb2} 26$ and $\mathrm{CuO}$ under atmospheric and high pressure. Thermochimica Acta 133 (0):73-77. doi:http://dx.doi.org/10.1016/0040-6031(88)87139-9

10. Giere EO, Brahimi A, Deiseroth HJ, Reinen D (1997) The Geometry and Electronic Structure of the Cu2+Polyhedra in Trirutile-Type Compounds Zn(Mg)1-xCuxSb2O6and the Dimorphism of CuSb2O6: A Solid State and EPR Study. Journal of Solid State Chemistry 131 (2):263-274

11. Mellor JW (1933) A Comprehensive Treatise on Inorganic and Theoretical Chemistry, vol IX: The Antimonious Acids and the Antimonites. Longmans, Green and Co., London

12. Ricco IMM, Focke WW, Conradie C (2004) Alternative oxidants for silicon fuel in time-delay compositions. Combustion Science and Technology 176 (9):1565-1575

13. Focke WW, Mkhize SS, Storey R, Fabbro OD, Muller E (2014) Facile synthesis of copper antimonite. Chemical Engineering Communications 201 (2):153-159

14. Powder Diffraction File (2006) International Centre for Diffraction Data.

15. Inorganic Crystal Structure Database (2012) FIZ Karlsruhe.

16. Coelho AA (2000) Whole-profile structure solution from powder diffraction data using simulated annealing. Journal of Applied Crystallography 33:899-908

17. Coelho AA (2003) Indexing of powder diffraction patterns by iterative use of singular value decomposition. Journal of Applied Crystallography 36:86-95

18. Le Bail AL, Y.; Jouanneaux, A. Structure determination by Powder Diffractometry. In: EPDIC7: 7th European Powder Diffraction Conference, Barcelona, 20-23 May 20002000.

19. Bergmann J, Le Bail A, Shirley R, Zlokazov V (2004) Renewed interest in powder diffraction data indexing. Zeitschrift fur Kristallographie 219 (12):783-790

20. Shankland K, David WIF, Sivia DS (1997) Routine ab initio structure determination of chlorothiazide by Xray powder diffraction using optimised data collection and analysis strategies. Journal of Materials Chemistry 7 (3):569-572

21. Von Dreele RB, Stephens PW, Smith GD, Blessing RH (2000) The first protein crystal structure determined from high-resolution X-ray powder diffraction data: A variant of T3R3 human insulin-zinc complex produced by grinding. Acta Crystallographica Section D: Biological Crystallography 56 (12):1549-1553

22. Lehmann CW, Stowasser $F(2007)$ The crystal structure of anhydrous $\beta$-caffeine as determined from X-ray powder-diffraction data. Chemistry - A European Journal 13 (10):2908-2911

23. AXS B (2011) DIFFRAC.TOPAS. . 4.1 edn.,

24. Laugier JB, B. (2002) LMGP-Suite of Programs for the interpretation of X-ray Experiments. Chekcell

ENSP/Laboratoire des Matériaux et du Génie Physique,, Saint Martin d'Hères, France

25. Evans JSO (2010) jEdit 4.3.1 for TOPAS. Material Science Forum 651:1

26. Anon (2012) Prediction of physical and chemical materials properties.

27. Anon (2010) MedeA Materials design and exploration analysis software. 2.5.17.2 edn. Materials Design, Inc,

28. Anon (2012) MedeA User's Guide. 2.4 edn.,

29. Kresse G, Joubert $D$ (1999) From ultrasoft pseudopotentials to the projector augmented-wave method. Physical Review B - Condensed Matter and Materials Physics 59 (3):1758-1775

30. Kresse G, Furthmüller J (1996) Efficient iterative schemes for ab initio total-energy calculations using a plane-wave basis set. Physical Review B - Condensed Matter and Materials Physics 54 (16):11169-11186

31. Kresse G, Furthmüller J (1996) Efficiency of ab-initio total energy calculations for metals and semiconductors using a plane-wave basis set. Computational Materials Science 6 (1):15-50

32. Parlinski K (2005) Software Phonon. Software PHONON.

33. Schutte CJH (1976) The Theory of Molecular Spectroscopy. The Quantum Mechanics and Group Theory of Vibrating and Rotating Molecules., vol 1. North-Holland, Amsterdam 
34. Schutte $\mathrm{CJH}$, Pretorius JA (2012) A computational study of the molecular and crystal structure and selected physical properties of octahydridosilasequioxane, (Si $2 \mathrm{O} 3 \mathrm{H} \mathrm{2}$ ) 4. II. Vibrational analysis. Proceedings of the Royal Society A: Mathematical, Physical and Engineering Sciences 468 (2139):851-870

35. Schutte $\mathrm{CJH}$, Pretorius JA (2011) A computational study of the molecular and crystal structure and selected physical properties of octahydridosilasequioxane-(Si2O 3H2)4. I. Electronic and structural aspects. Proceedings of the Royal Society A: Mathematical, Physical and Engineering Sciences 467 (2128):928-953 36. Schutte CJH (1973) Topics in current chemistry. The Infra-red Spectra of Crystalline Solids 36:57 37. Eby RK, Hawthorne FC (1993) Structural relations in copper oxysalt minerals. I. Structural hierarchy. Acta Crystallographica Section B 49 (1):28-56. doi:doi:10.1107/\$0108768192007274

38. Cooper MAH, F.C. (1995) The crystal structure of mottramite, and the nature of CU<-->Zn solid solution in the mottramite-descloizite series. The Canadian Mineralogist 33:1119-1124

39. Hawthorne PCBaFC (1995) Coordination-geometry structural pathways in $\mathrm{Cu}$ (super 2+) oxysalt minerals. The Canadian Mineralogist 33:889-905

40. Gavarri JR (1982) Evolution structurale d'oxydes isomorphes MeX2O4: Relation entre dilatation, vibrations et rigidite. Journal of Solid State Chemistry 43 (1):12-28

41. Gutierrez Puebla E, Gutierrez Rios E, Monge A, Rasines I (1982) Crystal growth and structure of diantimony(III) zinc oxide. Acta Crystallographica Section B 38 (7):2020-2022.

doi:doi:10.1107/S0567740882007791

42. Prokofiev AV, Ritter F, Assmus W, Gibson BJ, Kremer RK (2003) Crystal growth and characterization of the magnetic properties of CuSb2O6. Journal of Crystal Growth 247 (3-4):457-466.

doi:http://dx.doi.org/10.1016/S0022-0248(02)02062-6

43. Rebello A, Smith MG, Neumeier JJ, White BD, Yu YK (2013) Transition from one-dimensional antiferromagnetism to three-dimensional antiferromagnetic order in single-crystalline CuSb2O6. Physical Review B - Condensed Matter and Materials Physics 87 (22)

44. Gibson BJ, Kremer RK, Prokofiev AV, Assmus W, Ouladdiaf B (2004) Elastic neutron diffraction study of long-range antiferro-magnetic order in the $S=1 / 2$ quantum chain system CuSb2O6. Journal of Magnetism and Magnetic Materials 272-276:927-928

45. Yang CN, Yang CP (1966) One-dimensional chain of anisotropic spin-spin interactions. Physics Letters 20 (1):9-10

46. Yang CN, Yang CP (1966) One-dimensional chain of anisotropic spin-spin interactions. II. Properties of the ground-state energy per lattice site for an infinite system. Physical Review 150 (1):327-339

47. Yang CN, Yang CP (1966) One-dimensional chain of anisotropic spin-spin interactions. III. Applications. Physical Review 151 (1):258-264

48. Hatfield WE (1981) New magnetic and structural results for uniformly spaced, alternatingly spaced, and ladder-like copper (II) linear chain compounds (invited). Journal of Applied Physics 52 (3):1985-1990

49. Bonner JC (1978) One-dimensional model systems: Theoretical survey. Journal of Applied Physics 49

(3):1299-1304

50. Johnston DC, Kremer RK, Troyer M, Wang X, Klümper A, Bud'ko SL, Panchula AF, Canfield PC (2000)

Thermodynamics of spin $S=1 / 2$ antiferromagnetic uniform and alternating-exchange Heisenberg chains.

Physical Review B - Condensed Matter and Materials Physics 61 (14):9558-9606

\section{LIST OF TABLES}

Table 1 Details of X-ray powder data collection, crystallographic data and refinement for $\mathrm{cusb}_{2} \mathrm{O}_{4}$ 
Table 2 Fractional atomic coordinates and isotropic or equivalent displacement parameters

Table 3 The 62 PHONON modelled frequencies of the 84 normal modes (omega), multiplicity (M) and Raman (R), infrared (i) and irreducible representation (IR) of each mode

\section{LIST OF FIGURES}

Fig. 1 SEM micrograph of copper antimonite crystals.

Fig. 2 Powder diffraction patterns of the green $\mathrm{CuSb}_{2} \mathrm{O}_{4}$ (bottom) and $\mathrm{CuSb}_{2} \mathrm{O}_{6}$ the product obtained by heating it to $1100{ }^{\circ} \mathrm{C}$ (top). The symbols above the bottom pattern indicate major line positions of the impurity phase senarmontite $\left(\mathrm{Sb}_{2} \mathrm{O}_{3}\right)$.

Fig. $3 \mathrm{CuSb}_{2} \mathrm{O}_{4}$ structure representation as ball and stick (A) and with $\mathrm{CuO}_{6}$ polyhedra (B): projection along $c$ axis slightly rotated around $a$ and $b$.

Fig. 4 The $\mathrm{Cu}$ coordination polyhedra with $(\mathrm{A})$ interatomic distances $(\AA)$, and (B) bond angles $\left(^{\circ}\right)$.

Fig. 5 The Sb coordination polyhedra with $(\mathrm{A})$ interatomic distances $(\AA)$, and $(\mathrm{B})$ bond angles $\left(^{\circ}\right)$.

Fig. 6 Raman spectrum of the green $\mathrm{CuSb}_{2} \mathrm{O}_{4}$ obtained by subtracting the bands due to $\mathrm{Sb}_{2} \mathrm{O}_{3}$.

Fig. 7 (main panel) Semi-log plot of magnetic susceptibility of $\mathrm{CuSb}_{2} \mathrm{O}_{4}$. The line superimposed onto the data is a least-squares fit of a $1 \mathrm{D}$ Heisenberg $S=1 / 2$ model described in the text. (inset) Magnetization at the two extremes of temperatures of this study. 
Fig. 8 Magnetic susceptibility of $\mathrm{CuSb}_{2} \mathrm{O}_{4}$ towards low temperatures, in three values of applied magnetic field. Arrows mark the location of a maximum susceptibility.

Fig. 9 (a) Specific heat of $\mathrm{CuSb}_{2} \mathrm{O}_{4}$. The phase transition at $T_{\mathrm{m}}$ is marked by an arrow. (inset) Specific heat $v s . T^{2}$ provides assessment of the $T \rightarrow 0$ electronic specific heat as discussed in the text. (b) Total entropy of $\mathrm{CuSb}_{2} \mathrm{O}_{4}$. An inflection point marked by an arrow corresponds to the phase transition at $T$. 


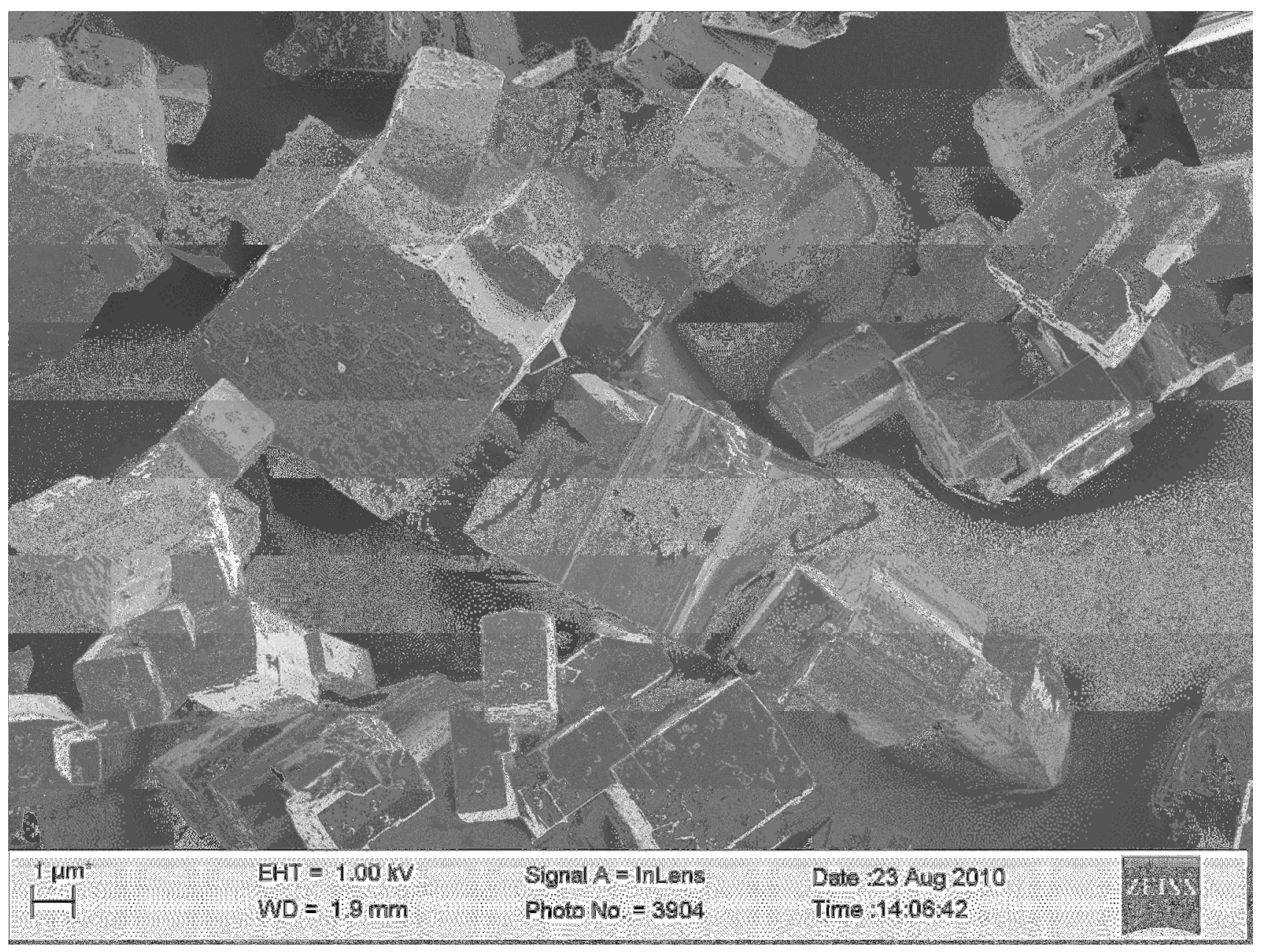

Fig. 1 SEM micrograph of copper antimonite crystals. 


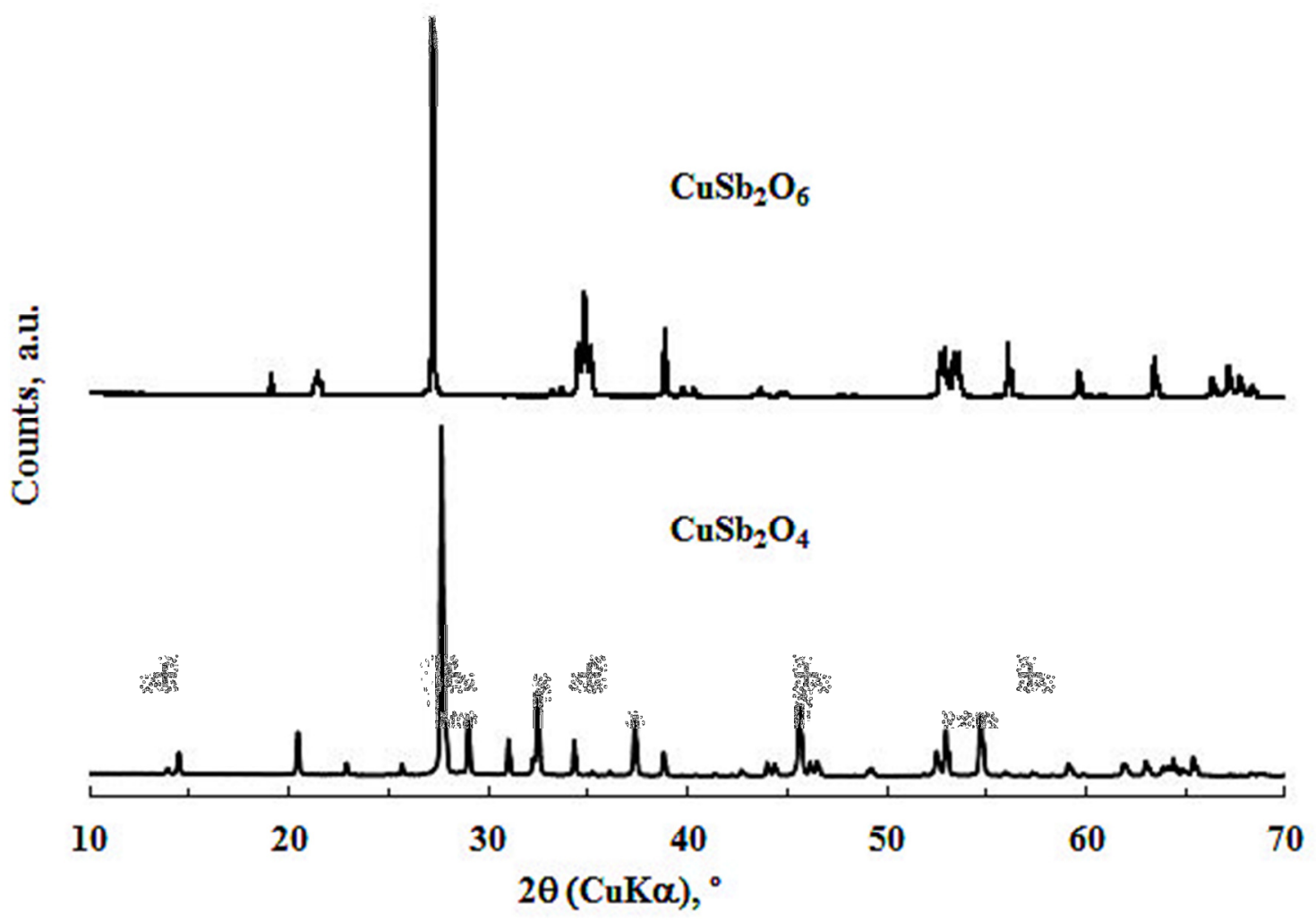

Fig. 2 Powder diffraction patterns of the green $\mathrm{CuSb}_{2} \mathrm{O}_{4}$ (bottom) and $\mathrm{CuSb}_{2} \mathrm{O}_{6}$ the product obtained by heating it to $1100{ }^{\circ} \mathrm{C}$ (top). The symbols above the bottom pattern indicate major line positions of the impurity phase senarmontite $\left(\mathrm{Sb}_{2} \mathrm{O}_{3}\right)$. 


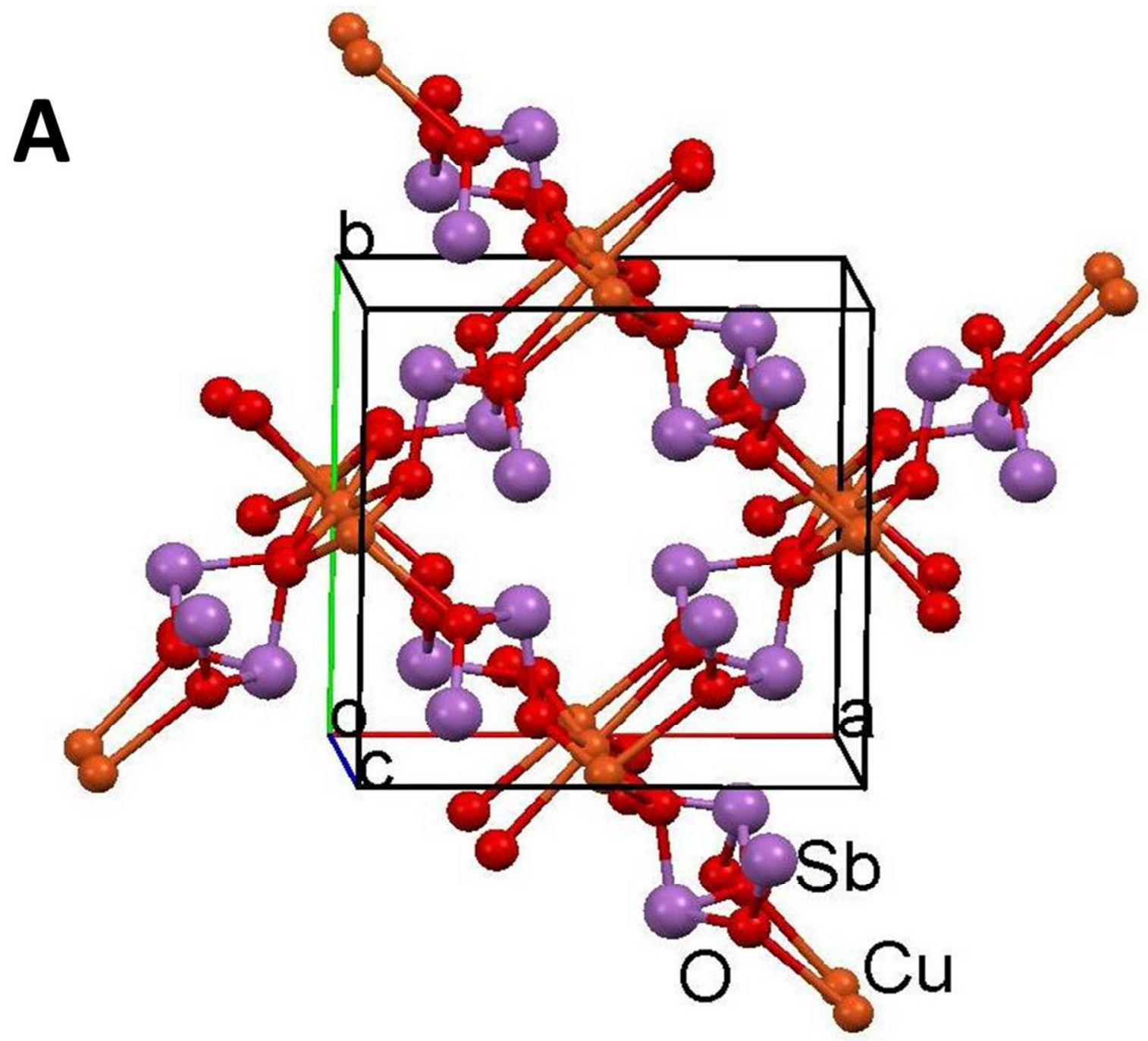

Fig. $3 \mathrm{CuSb}_{2} \mathrm{O}_{4}$ structure representation as ball and stick (A) and with $\mathrm{CuO}_{6}$ polyhedra (B): projection along $c$ axis slightly rotated around $a$ and $b$. 


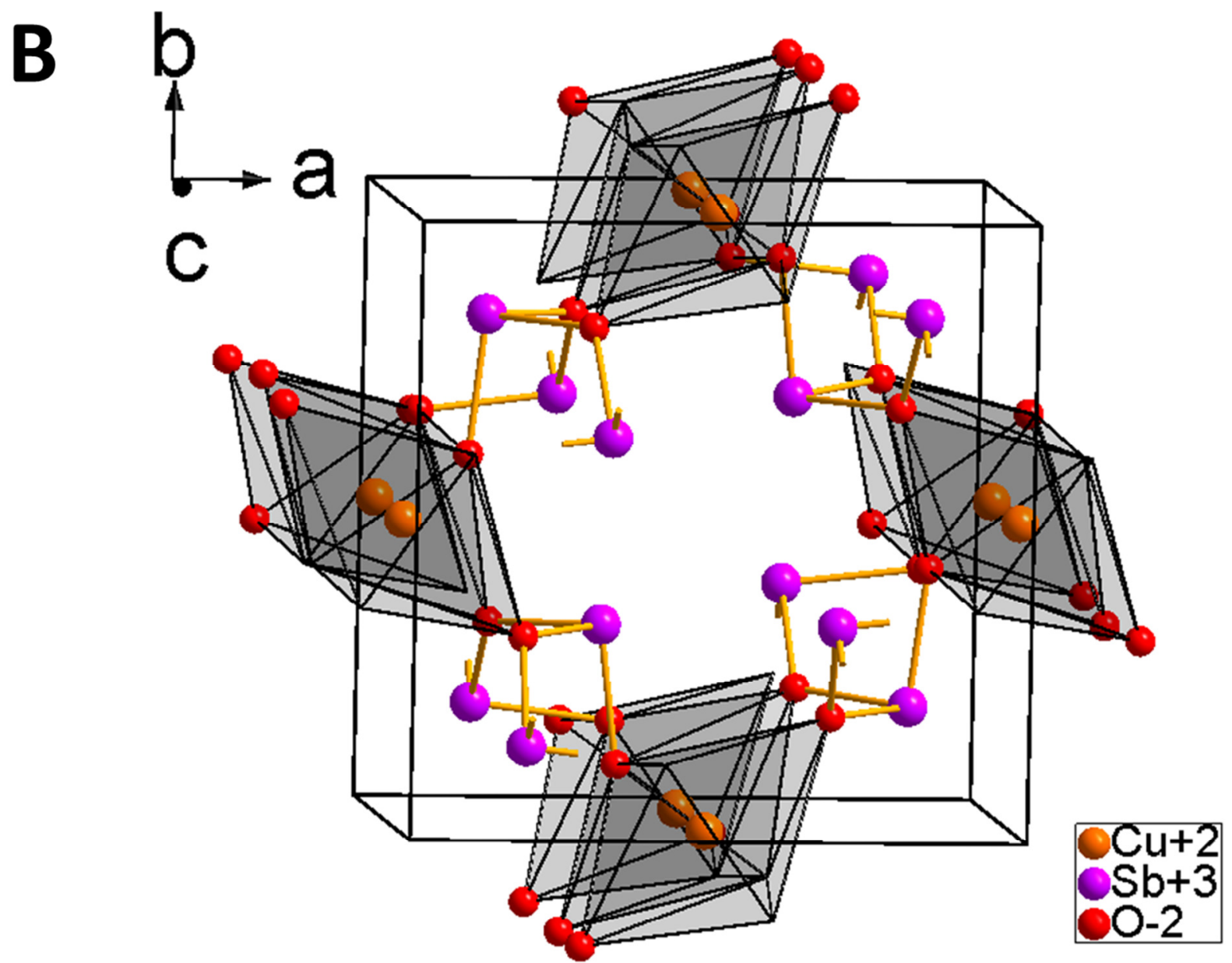

Fig. $3 \mathrm{CuSb}_{2} \mathrm{O}_{4}$ structure representation as ball and stick (A) and with $\mathrm{CuO}_{6}$ polyhedra (B): projection along $c$ axis slightly rotated around $a$ and $b$. 
A

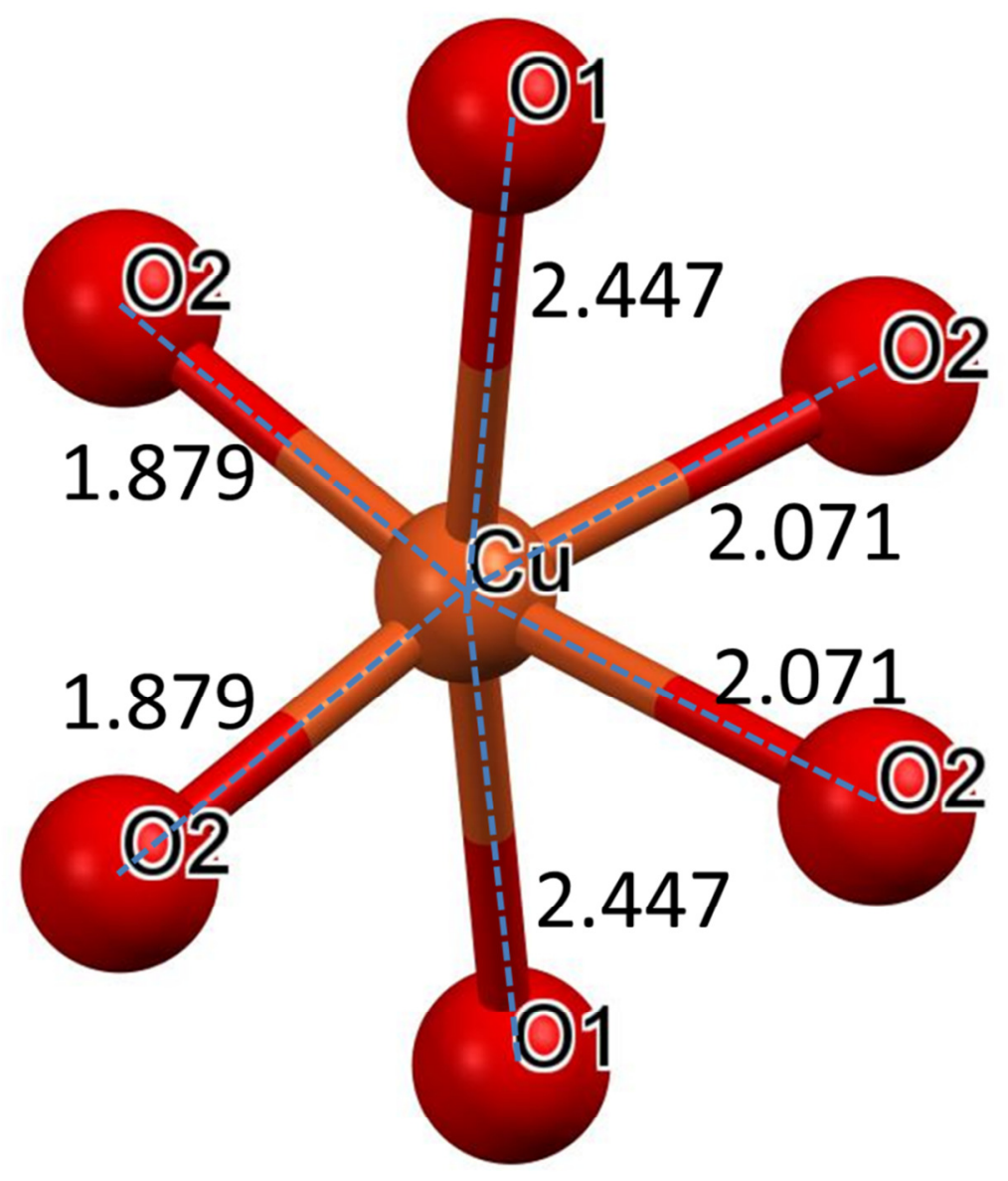

Fig. 4 The $\mathrm{Cu}$ coordination polyhedra with $(\mathrm{A})$ interatomic distances $(\AA)$, and $(\mathrm{B})$ bond angles $\left({ }^{\circ}\right)$. 


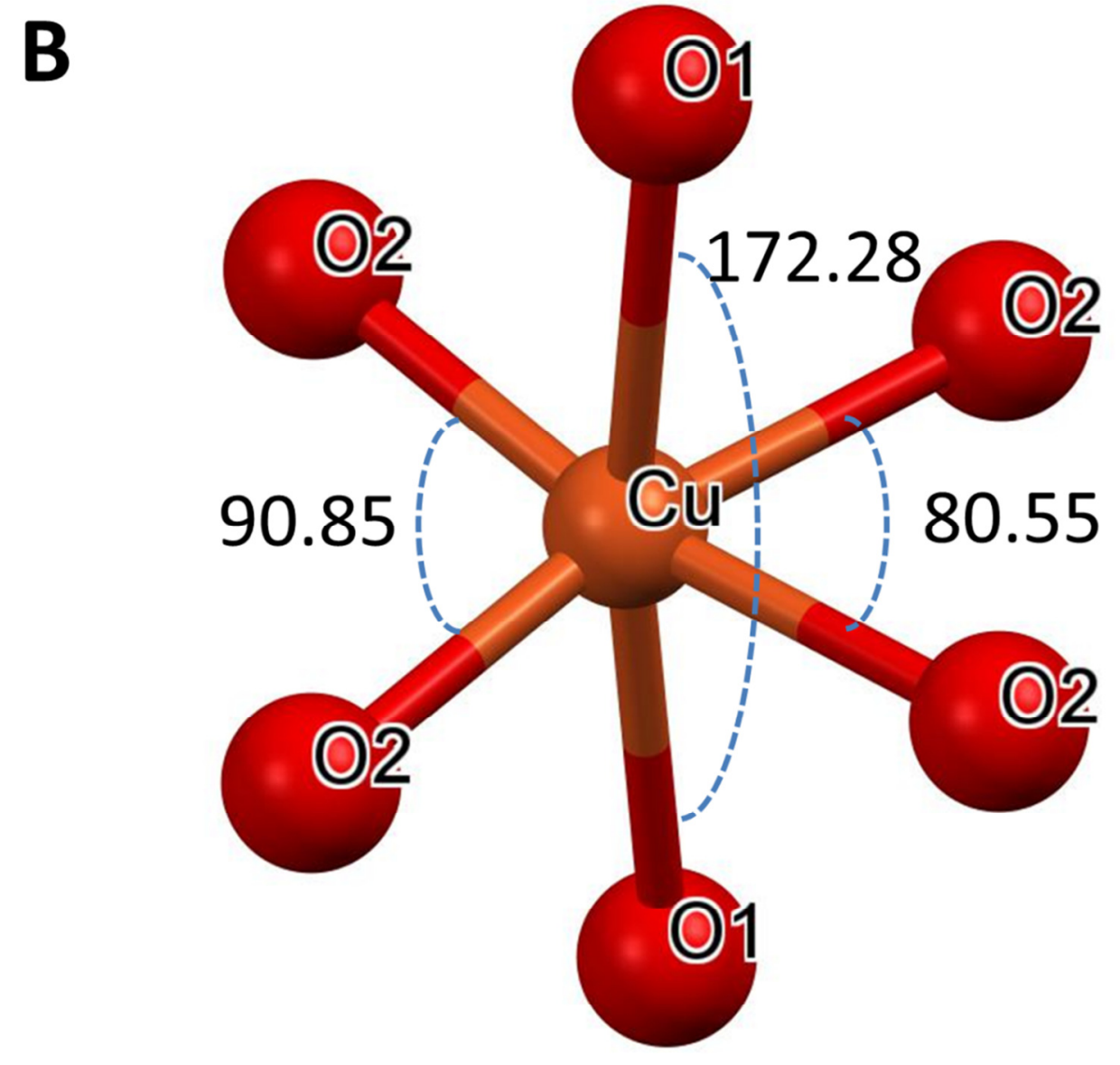

Fig. 4 The $\mathrm{Cu}$ coordination polyhedra with $(\mathrm{A})$ interatomic distances $(\AA)$, and (B) bond angles $\left({ }^{\circ}\right)$. 


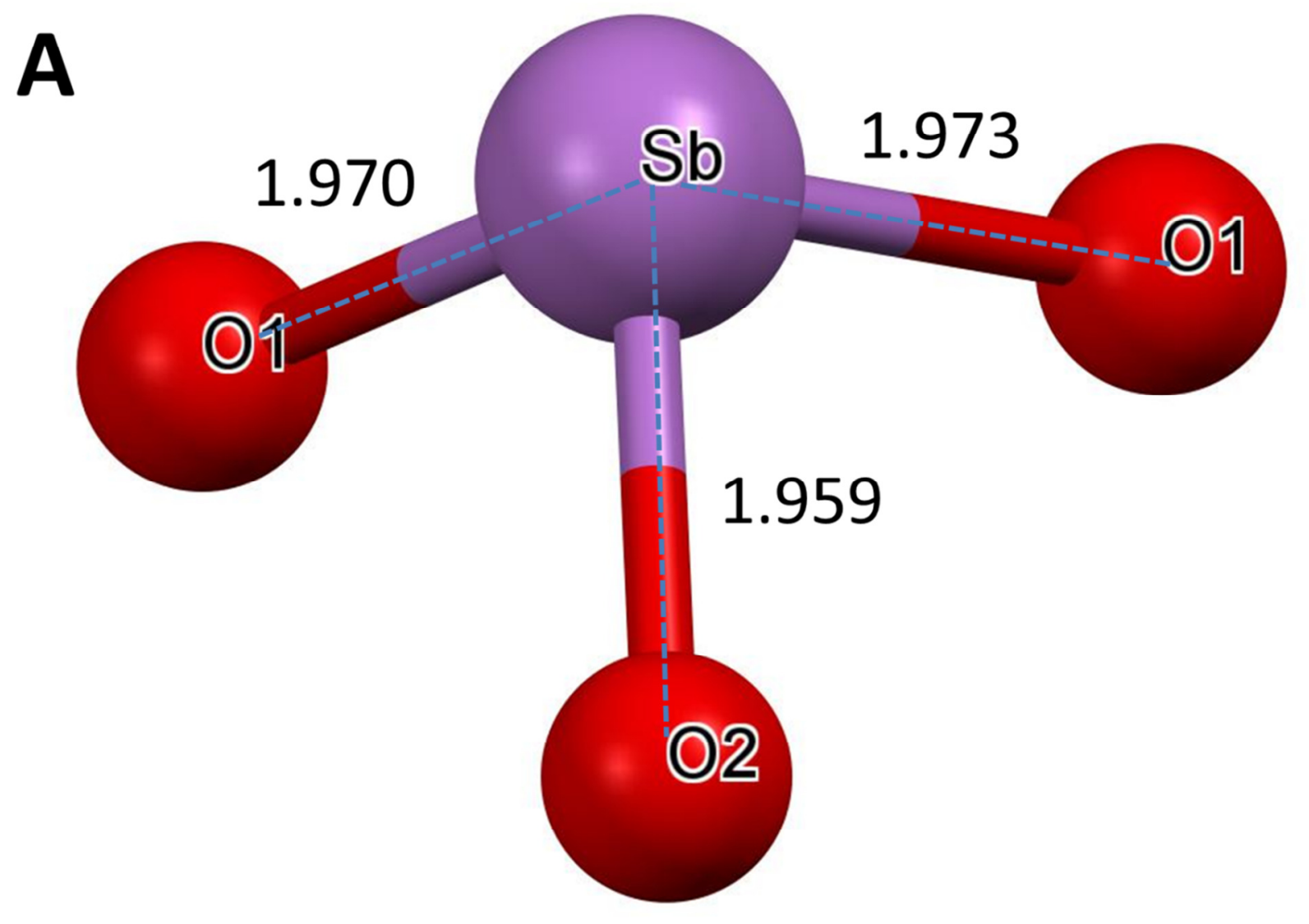

Fig. 5 The Sb coordination polyhedra with (A) interatomic distances $(\AA)$, and (B) bond angles $\left(^{\circ}\right)$. 


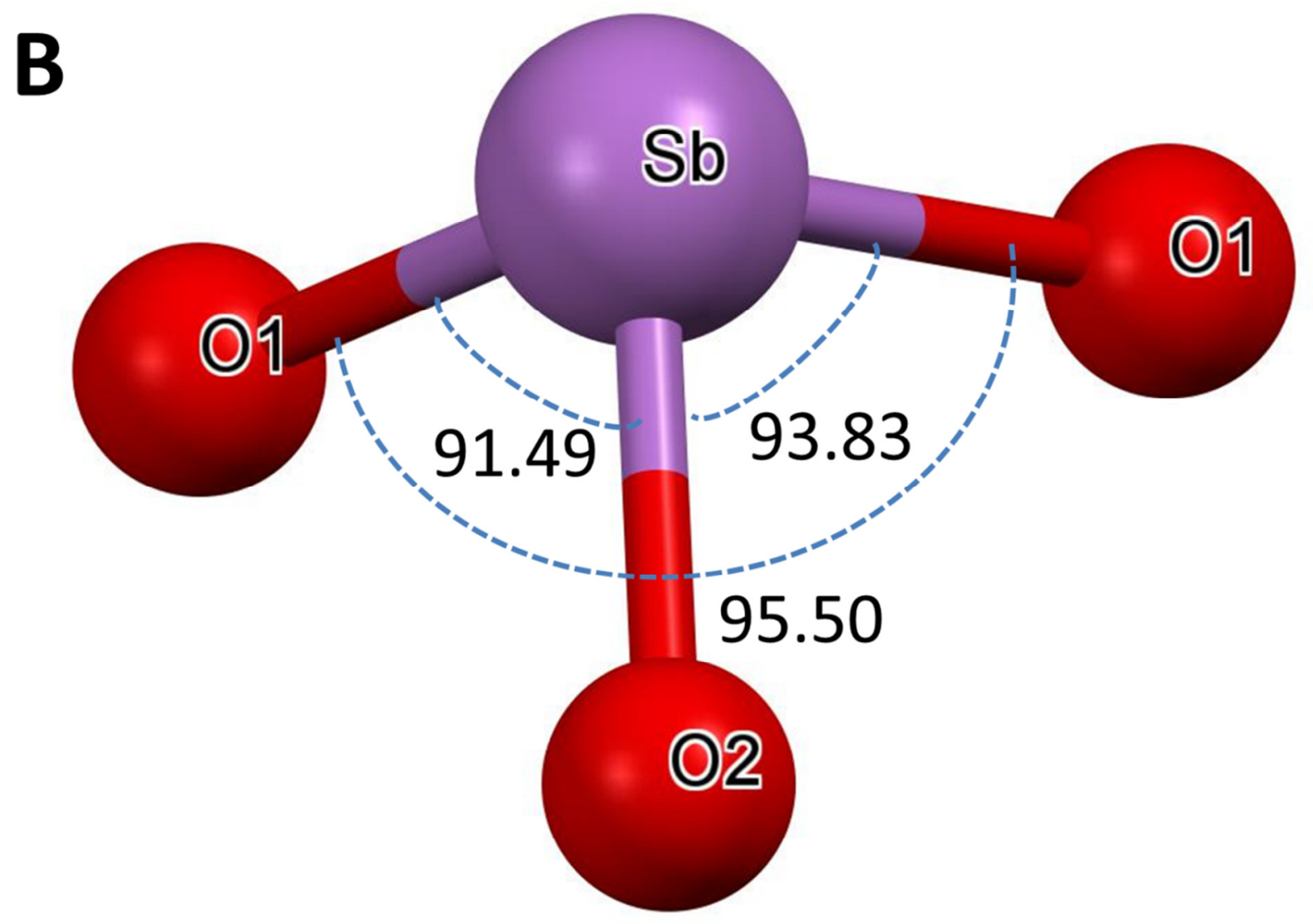

Fig. 5 The Sb coordination polyhedra with (A) interatomic distances $(\AA)$, and (B) bond angles $\left(^{\circ}\right)$. 


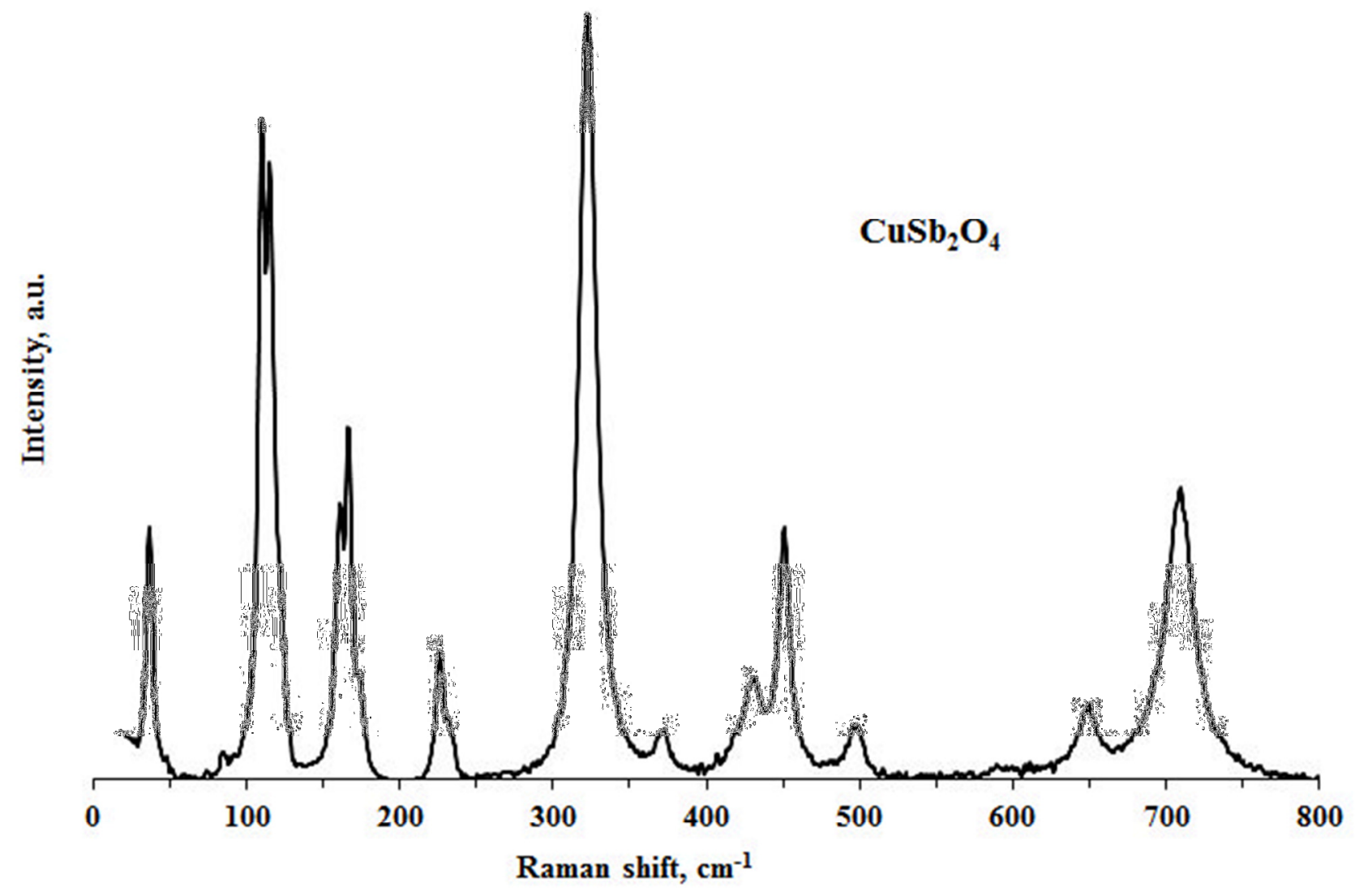

Fig. 6 Raman spectrum of the green $\mathrm{CuSb}_{2} \mathrm{O}_{4}$ obtained by subtracting the bands due to $\mathrm{Sb}_{2} \mathrm{O}_{3}$. 


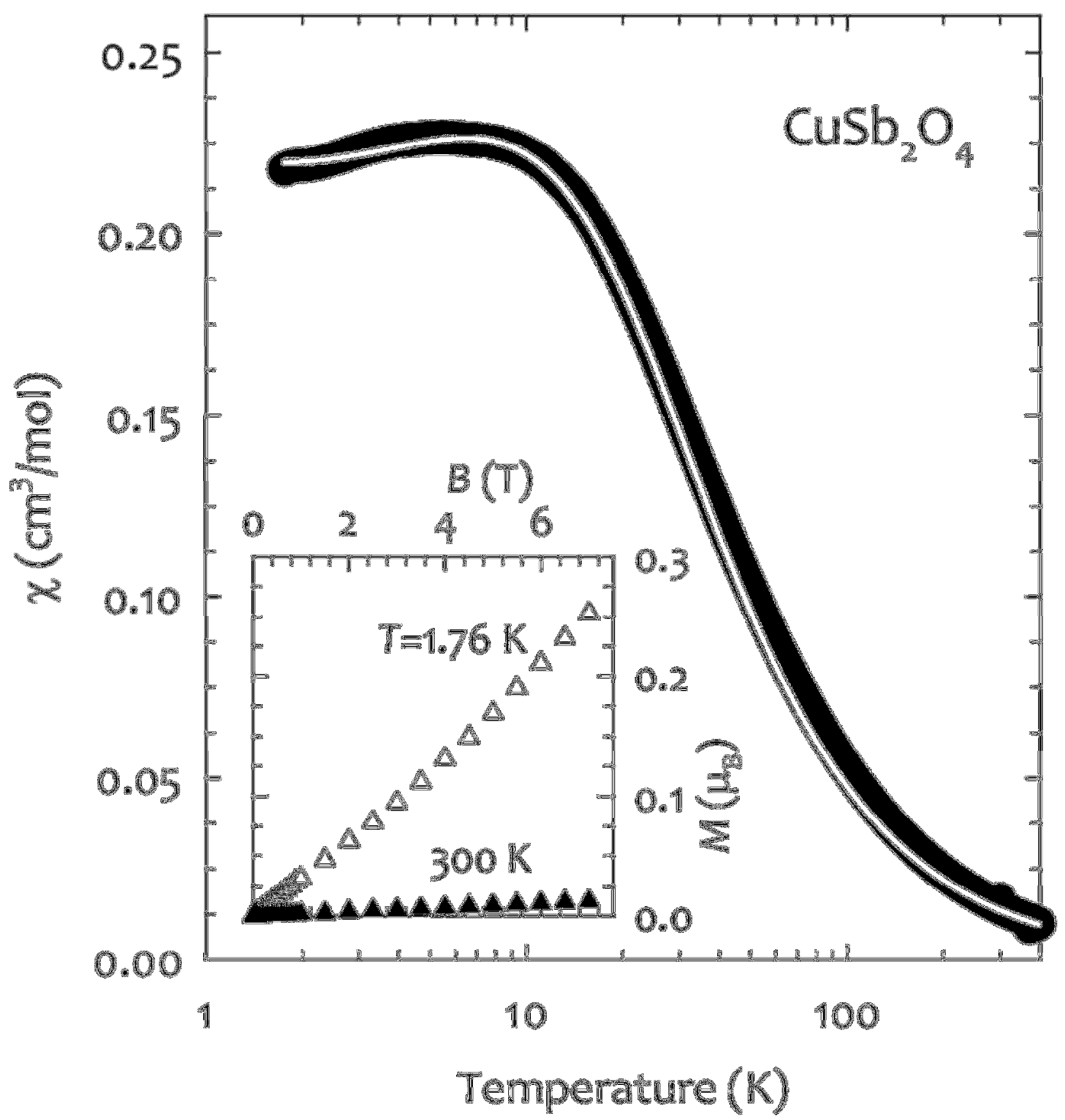

Fig. 7 (main panel) Semi-log plot of magnetic susceptibility of $\mathrm{CuSb}_{2} \mathrm{O}_{4}$. The line superimposed onto the data is a least-squares fit of a 1D Heisenberg $S=1 / 2$ model described in the text. (inset) Magnetization at the two extremes of temperatures of this study. 


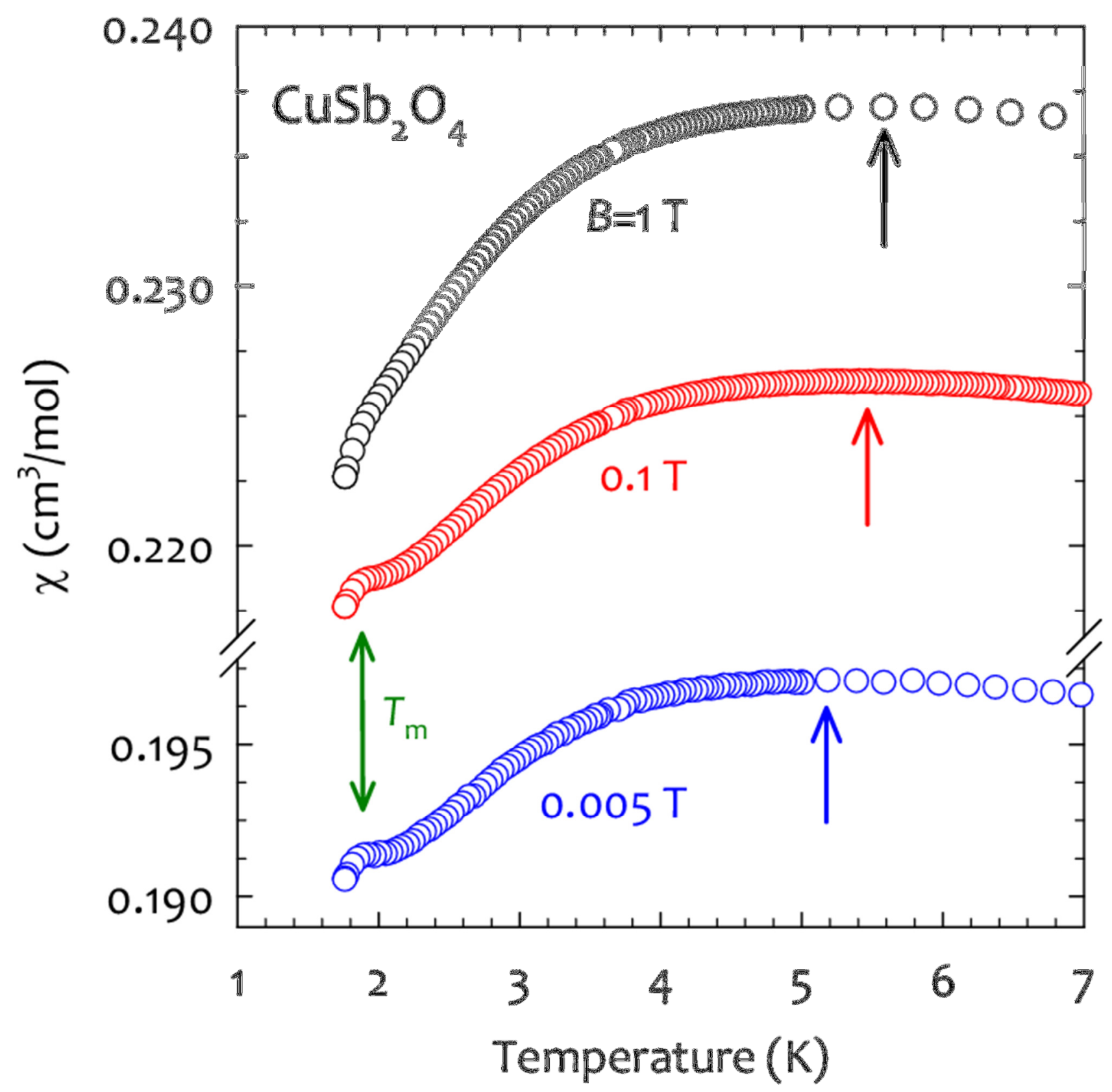

Fig. 8 Magnetic susceptibility of $\mathrm{CuSb}_{2} \mathrm{O}_{4}$ towards low temperatures, in three values of applied magnetic field. Arrows mark the location of a maximum susceptibility. 


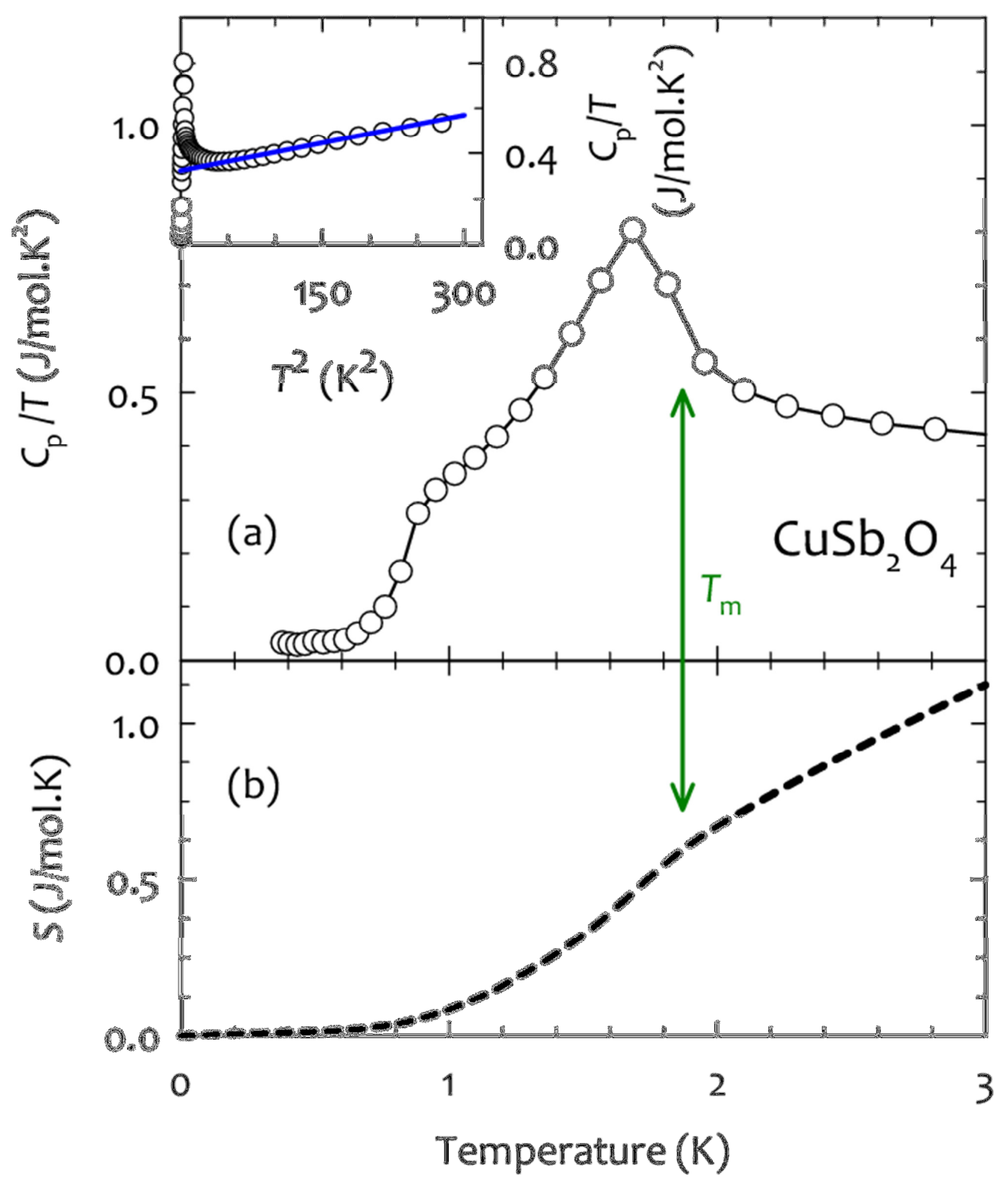

Fig. 9 (a) Specific heat of $\mathrm{CuSb}_{2} \mathrm{O}_{4}$. The phase transition at $T_{\mathrm{m}}$ is marked by an arrow. (inset) Specific heat vs. $T^{2}$ provides assessment of the $T \rightarrow 0$ electronic specific heat as discussed in the text. (b) Total entropy of $\mathrm{CuSb}_{2} \mathrm{O}_{4}$. An inflection point marked by an arrow corresponds to the phase transition at $T$. 